\title{
Long Period Gratings in New Generation Optical Fibers
}

\author{
Agostino Iadiciccoㄹ, Domenico Paladino², Pierluigi Pilla ${ }^{2}$ \\ Stefania Campopiano ${ }^{1}$, Antonello Cutolo ${ }^{2}$ and Andrea Cusano ${ }^{2}$ \\ 'Department for Technologies, University of Naples "Parthenope" \\ ${ }^{2}$ Optoelectronic Division-Engineering Department, University of Sannio \\ Italy
}

\section{Introduction}

The development of fiber gratings has had a significant impact on research and development in telecommunications and fiber optic sensing. Fiber gratings are intrinsic devices that allow control over the properties of light propagating within the fiber - they are used as spectral filters, as dispersion compensating components and in wavelength division multiplexing systems (Erdogan, 1997). The sensitivity of their properties to perturbation of the fiber by the surrounding environmental conditions has led to extensive study of their use as fiber sensor elements (Kersey et al., 1997). Fiber gratings consist of a periodic perturbation of the properties of the optical fiber, generally of the refractive index of the core and/or geometry, and fall into two general classifications based upon the period of the grating. Short-period fiber gratings, or fiber Bragg gratings (FBGs), have a sub-micron period and act to couple light from the forward-propagating core mode of the optical fiber to a backward, counter-propagating one (Kashyap, 1999; Canning, 2008; Cusano et al., 2009a). The long-period gratings (LPGs), instead, have period typically in the range 0.1-1 $\mathrm{mm}$ (James \& Tatam, 2003). The LPG promotes coupling between the propagating core mode and co-propagating cladding modes. The high attenuation of the cladding modes results in the transmission spectrum of the fiber containing a series of attenuation bands centred at discrete wavelengths, each attenuation band corresponding to the coupling to a different cladding mode. The exact form of the spectrum, and the centre wavelengths of the attenuation bands, are sensitive to the period of the LPG, the length of the LPG and to the local environment: temperature, strain, bend radius and the refractive index (RI) of the medium surrounding the fiber. The peculiar spectral features of LPGs made them broadly used in many applications ranging from telecommunications to sensing (Bhatia, 1999). In particular, LPGs represent above all one of the most promising fiber grating technological platforms, to be employed in a number of chemical applications because of their intrinsic sensitivity to surrounding RI (SRI) changes (Shu et al., 2002). Up to now great efforts have been done in order to enhance the performance of LPGs in single mode fibers (SMFs) in terms of tuning capability and/or sensitivity. For instance several approaches have been proposed to achieve remarkable sensitivities such as cladding etching, LPG design for coupling to higher order modes near their dispersion turning points or in-fiber complex 
configuration including multi-gratings (Chung \& Yin, 2004; Iadicicco et al., 2007, 2008; Pilla et al., 2008). Additionally, once the effects of depositing a thin high RI (HRI) layer onto the cladding over the grating region have been discovered, huge sensitivity enhancements in comparison to bare LPGs have been obtained due to the so-called modal transition (Del Villar et al., 2005; Cusano et al., 2005, 2006a).

On the other side, it is worth noting that new fiber designs such as D-shaped fibers and photonic crystal fibers (PCFs) (with solid and air core) capable to offer new perspective in sensing and telecommunications applications have attracted the attention of several researcher groups and scientists (Tseng \& Chen, 1992; Smith et al., 2004; Gordon et al., 2007; Kaiser et al., 1974; Knight et al., 1996). However to increase the impact of the new generation fibers technology, in-fiber components such as grating filters are required. In this chapter the recent progresses of LPGs into new generation fibers will be reported. Fabrication techniques and novel applications fields offered by the hosting fiber will be discussed. In particular here the following optical fiber designs will be take into considerations:

i. D-shaped fiber. This category refers to a generic optical fiber showing a D-shaped transversal section. In such a fiber the core can be very close to the flat side of the " $\mathrm{D}$ " shape. This proximity allows access to the core electromagnetic fields more easily than in standard SMF and thus D-fiber is extremely attractive especially in sensing applications. A D-shaped fiber can be readily obtained from a standard SMF by sidepolishing (Tseng \& Chen, 1992) or, alternatively, is commercially available from KVH Industries, Inc. (Smith et al., 2004; Gordon et al., 2007). The possibility to combine LPGs with D-fibers has represented and still represents an open challenge for the scientific community. In this kind of fiber LPGs can be achieved impressing physical modification of the core (intra-core LPGs). Alternatively, thanks to the proximity of the core region to the flat surface, periodic modification of the effective RI of the core mode (forming the LPG) can be induced via evanescent-wave interaction if the flat surface is morphologically modified with appropriate pitch (Jang et al., 2009). Here fabrications and applications of both D-fiber LPGs are resumed.

ii. Photonic crystal fibers. They refer to a new class of optical fibers that have wavelengthscale morphological microstructure running down their length (Knight et al., 1996). They, according to their guiding mechanisms, may be divided into index-guiding PCFs and photonic band-gap fibers (PBFs). The former permit light to be guided in silica solid core while the second one enable the light guiding in the air core. Even if the first PCF was proposed in 1974 (Kaiser et al., 1974), the first pure silica PCF was achieved for practical use in the middle of the 1990s (Birks et al., 1995; Knight et al., 1996). Such structured optical fibers, indeed, thanks to their composite nature enable a plenty of possibilities and functionalities hitherto not possible - long range spectroscopy as well as large mode areas fiber laser just to name a few (Canning, 2008). Particular attention has been focused on hollow core PCFs (HC-PCFs) due to the lattice assisted light propagation within the hollow core (Smith et al., 2003). This particular feature, indeed, has a number of advantages such as lower Rayleigh scattering, reduced nonlinearity, novel dispersion characteristics, and potentially lower loss compared to conventional optical fibers. Fabrication of gratings in PCFs fiber still represents a challenge for the scientific community (Cusano et al, 2009; Y. Wang, 2010). Here the fabrication of LPGs in PCFs as well as the novel application fields offered by the hosting fiber will be discussed. 
The next sections are organized as follows: Section 2 provides a brief review of LPGs in SMFs, Section 3 focuses on LPGs in D-shaped fibers and Section 4 reports recent progresses about LPGs in PCFs.

\section{Long period gratings: a view back}

Long Period Gratings are a periodic perturbation of the properties of the optical fiber, generally of the refractive index of the core and/or geometry, in a single mode fiber. They have periods typically ranging between $200 \mu \mathrm{m}$ and $500 \mu \mathrm{m}$ and lengths around $2-3 \mathrm{~cm}$. The perturbation acts on the fundamental core mode enabling power transfer to a discrete set of co-propagating cladding modes that are excited at different wavelengths where a phase matching condition is satisfied. This modal coupling process determines a loss in the core mode that is reflected into a series of attenuation bands in the transmission spectrum of the optical fiber. Although they were primarily introduced as devices for optical communications (Vengsarkar et al., 1996), for which they have been used to develop band rejection filters, gain equalizers, optical amplifiers, fiber couplers, dispersion compensators (Chiang \& Liu, 2006), however, they have immediately found vast application in the sensing field (Bathia et al., 1996). In fact any physical entity able to affect the difference of the core and cladding effective indices and/or the grating period and length, results in a change of the transmission spectrum in terms of central wavelength, depth and bandwidth of attenuation bands. Therefore LPGs have been investigated as sensors for a number of environmental parameters such as temperature, strain, bending and ambient RI (James \& Tatam, 2003). LPGs are classically realized by exposing an optical fiber to UV lasers through an amplitude mask and exploiting the photosensitivity of silica glass. In this regard, despite extensive research on the physical mechanisms underlying the fiber photosensitivity in the past decades, there are some aspects that are not fully understood. The reason is that a number of mechanisms take part in this optical phenomenon, sometimes simultaneously, whose relative weight depends on the specific chemical composition of the fiber and drawing process, the photosensitization (hydrogen loading, flame brushing, co-doping, strain) and writing processes (irradiation power, wavelength, duration) (Vasiliev et al., 2005). The amplitude mask is usually made of a chrome- plated silica substrate that is patterned in order to have light transmitting slits alternating with reflective regions. The fiber is placed within a few millimetres behind the amplitude mask with its axis oriented perpendicular to the mask slits. A cylindrical lens focuses the Gaussian spot of the laser into a line parallel to the fiber axis. The UV light passing through the amplitude mask imprints a RI modulation onto the photosensitive fiber core thus yielding a grating with the same period as that of the mask pattern. The shortcomings of the amplitude mask technique are the restrictions on the grating period and length that are fixed by the geometrical features of the mask itself. Moreover amplitude masks can be easily damaged if they are exposed to UV light whose intensity exceeds their damage threshold thus requiring long exposure times at limited source intensities. Another widespread grating inscription method is the point-by-point writing technique, in which the grating is obtained by focusing the laser source in a single spot on the fiber and successively displacing the fiber of the required grating periodicity to induce the next index change. This method is far more flexible than the amplitude mask because length and grating index profiles are fully reconfigurable. On the other side, the former allows the grating to be written all at once and offers more precision in the spectral response which is critical for some devices such as cascaded long-period gratings. 
The most frequently employed UV laser wavelengths for LPGs fabrication are 248nm and 193nm (from $\mathrm{KrF}$ and $\mathrm{ArF}$ excimer lasers, respectively) where are located strong absorption peaks due to defects of the $\mathrm{GeO}_{2}-\mathrm{SiO}_{2}$ network. Ultra-short wavelength of $152 \mathrm{~nm}$ from an $\mathrm{F}_{2}$ laser was used to produce LPGs in SMF-28 without prior hydrogen loading. The inscribed gratings, being immune to the problem of post-writing hydrogen out-diffusion, showed higher thermal stability compared to those realized by the standard photosensitizing technique. Recently, high intensity femtosecond laser pulses at longer wavelengths $(211 \mathrm{~nm}$, $264 \mathrm{~nm}$ and even $800 \mathrm{~nm}$ ) are becoming a fairly widespread method to induce RI modulation through a multi-photon absorption process that does not necessarily require photosensitization (Kalachev et al., 2005). Even if the UV writing methods by means of an amplitude mask or through a point-by-point process are the most commonly and readily used writing methods in research and industry, they have certain general shortcomings: a large number of masks is required to fabricate gratings with different periods; photosensitizing pre-treatments are necessary to facilitate the RI change; UV written gratings generally suffer poor thermal stability; last but not least, UV laser sources are expensive. For these reasons several non-photosensitive techniques for grating fabrication have been investigated. In this regard, refractive index modulation produced by high temperature thermal treatments exploiting $\mathrm{CO}_{2}$ lasers $(10.6 \mu \mathrm{m}$ wavelength) or an electric arc discharge has received great attention in last years. Both methods, to obtain the localized heating of the fiber, rely on a point-by-point writing approach and therefore they inherit the advantageous flexibility already mentioned. Also for these techniques there are several mechanisms that contribute to the refractive index modulation: relaxation of frozen stresses during fiber drawing, physical deformation, glass compaction or expansion, core dopants diffusion, among which the predominant cause depends on the heating treatment, the fiber type and any mechanical stress applied (Rego et al., 2005a; Y. Wang, 2010). For example, the arc discharge technique was used to form LPGs into pure-silica PCFs without any physical deformation (e.g. air holes collapse) by exploiting the glass structure change. The refractive index modulation was attributed to a glass density reduction due to the rapid heatingcooling process (Morishita \& Miyake, 2004). It is worth to observe that an additional benefit of the electric arc technique lie in the fact that it is based on a very simple fabrication procedure needing inexpensive equipment. However it should be also pointed out as a major pitfall of these techniques that the intrinsic asymmetry in the heating process leads to birefringence with consequent polarization dependent losses or coupling to azimuthally asymmetric cladding modes (Rego et al., 2006).

Coming back to the applications of LPGs as sensors we can identify four physical parameters of interest: applied tensile stress can modify the effective indices of core and cladding modes through the elasto-optic effect and the grating period because of elongation ; thermo-optic effect is responsible for the effective index change while thermal expansion for period modification in the case of the temperature changes (Shu et al., 2002); bending breaks the cylindrical symmetry of the waveguide promoting coupling to azhymuthally asymmetric cladding modes that are differently affected in their effective indices depending on the region of the fiber where they are confined (Block et al., 2006); finally the effective indices of cladding modes directly depend on the index contrast between the cladding and the surrounding medium being a boundary condition in the solution of the waveguide equation (Patrick et al. 1998). An interesting feature of LPGs is that the sensitivity to a particular measurand depends drastically on the order of the coupled cladding mode and on the type of the fiber. This makes possible the discrimination of different parameters 
acting simultaneously on the sensor and offers the possibility to design devices that are particularly sensitive or insensitive to a given stimulus (Bathia, 1999). LPGs written in standard optical fibers offer a temperature sensitivity up to one order of magnitude larger than FBGs and strain sensitivity to almost double by appropriate choice of observed cladding mode. Altering the fiber composition to increase the difference in the thermo-optic coefficients of core and cladding can be a valuable means to achieve higher sensitivities up to $2.75 \mathrm{~nm} /{ }^{\circ} \mathrm{C}$ (Shu et al., 2001). Among all, one of the most appealing features of LPGs is their intrinsic sensitivity to changes of the SRI because it can serve as a basis for achieving biomolecular and chemical sensors. The first applications of this feature, however, were more like solution concentration sensors since the bare LPG alone does not possess any chemical selectivity (Falciai et al., 2001; Falate et al., 2005). The deposition of thin overlay materials that can change their RI as a consequence of a physic-chemical interaction with the surrounding environment has opened a very interesting niche of applications (De Lisa et al., 2000). Moreover, another major pitfall for bare LPGs is their scarce SRI sensitivity in low index ambient (air, water) while they show maximum sensitivity for SRIs close to the cladding RI, typically around 1.45. In this context, a paradigm shift has been represented by the integration of nano-scale polymer overlays with HRI than the cladding and by the discovery of the modal transition phenomenon (Rees et al., 2002; Del Villar et al., 2005; Z. Wang et al. 2005; Cusano et al., 2005). It is by now very well known that the SRI sensitivity of LPGs can be optimized for the specific measurement environment through the deposition of a HRI thin film by acting on its thickness (ranging in hundreds of nanometres). Sensitivities as high as thousands of nanometres for a unitary change of SRI can be easily obtained and therefore LPGs coated by HRI functional layers have been successfully exploited for chemical and biomolecular sensing (Cusano et al., 2006b; Pilla et al., 2009). Humidity sensing is a fairly investigated application, that was performed with LPGs coated by thin films of different hygrosensitive materials (Tan et al., 2005; Kostantaki et al., 2006; Liu et al., 2007; Venugopalan et al., 2008). A zeolite overlay was used in combination with LPGs to detect the presence of few ppm of toluene and isopropanol vapours (Zhang et al., 2008). A sol-gel derived coating of tin dioxide with optimized thickness for high sensitivity $(\approx 200 \mathrm{~nm})$ was used to detect ethanol vapours claiming a resolution of $1 \mathrm{ppm}$ (Gu et al., 2006). A partially etched LPG with cladding substituted by a polymer coating of finely tuned RI and able to perform solid-phase microextraction of organic solvents such as xylene, cyclohexane and gasoline was demonstrated. The extra peculiarity of this study being the interrogation system potentially highly miniaturizable and based on the concept of the cavity ring down spectroscopy (Barnes et. al, 2010). A very sensitive probe for $\mathrm{pH}$ was manufactured by means of electrostatically self assembled multilayers without the use of colorants. The transduction principle was the swelling of the overlay as a consequence of increased concentration of hydrogen ions (Corres et al., 2007).

In the never ending quest for increased SRI sensitivity, a growing interest, both theoretical and experimental, has been recently shown also for the possibility to excite surface plasma waves by means of cladding modes (Tang et al., 2006, He et al., 2006). A Pd-coated LPG was used as hydrogen sensor (Wei et al., 2008). A particular dispersion behaviour of one of the cladding modes obtained for a specific grating period at a certain wavelength, the so-called turn around point (TAP), together with functional coatings of synthetic or biological nature, was exploited to obtain ultra-sensitivity for volatile organic compounds or biomolecules detection (Chen et al., 2007; Z. Wang et al., 2009; Topliss et al., 2010). It should be noted that the number of biosensing applications with LPGs is rapidly growing and it can be foreseen 
that it will represent an area of major interest in coming years (Eggen et al., 2010; Smietana et al., 2011). In this context LPGs realized in Photonic Crystal Fibers are extremely attractive for the possibility to achieve very intense light matter interactions with a unique optofluidic design and with nano-liter sample consumption (Rindorf et al.,2006; He et al., 2011). However, the attractiveness of the SRI sensitivity of LPGs is not limited to the field of sensing applications and it is extended to the optical communication domain for the possibility to develop tuneable filters and optical modulators (Yin et al., 2001; Chung et al., 2004; J. Lee et al., 2007). An interesting and relatively new trend in LPGs made in classical telecom fibers is the fabrication of compound structures characterized by spectral details of finer scale for higher resolution in the measurements of environmental parameters or to obtain a compensation against cross-sensitivities (D. Kim et al, 2006; Pilla et al., 2008; Jiang et al.,2009; Mosquera et al., 2010) .

\section{Long period gratings in D-shaped fibers}

The main advantage of LPGs over short-period FBGs is their intrinsic SRI sensitivity. Nevertheless, the fiber section geometry strongly influences the sensitivity characteristics of the considered LPG, in terms of SRI as well as in terms of the other external parameters able to induce changes in the grating spectrum: temperature, strain, bending, etc. In general, one of the most obvious manner to increase the interaction of the light propagating within an optical fiber and the surroundings is represented by the reduction of their distance from the core layer. The fiber structure that better satisfies such a need avoiding the microstructuring of the fiber itself is the D-shaped optical fiber. In such a fiber the core can be very close to the flat side of the " $\mathrm{D}$ " shape maintaining a certain robustness of the fiber structure, especially if compared with a SMF uniformly thinned - preserving its azimuthal symmetry - to reach the same distance of the core from the surroundings. Obviously, a first type of Dshaped fiber can be readily obtained from a standard SMF (see Fig. 1(a)) by side-polishing (Tseng \& Chen, 1992). On the other hand, a special D-shaped fiber is commercially available from KVH Industries, Inc.: it is a polarization maintaining SMF. Such a structure has been successfully exploited in the past for applications in both telecommunications and sensing (Smith et al., 2004, 2006; Smith, 2005; Gibson et al., 2007; Gordon et al., 2007). Note that slightly different geometrical features have been reported for this D-fiber by the different research groups involved with it in the past. However, Fig. 1 tries to compare the transversal geometrical features of a standard SMF with those of the D-fiber supplied by $\mathrm{KVH}$. Differently from the standard SMF, the D-fiber is a three-layer structure. In particular, it presents an elliptical Ge-doped core (major and minor axis of $\sim 5$ and $\sim 2.5 \mu \mathrm{m}$, respectively, and RI of 1.4756) with the major axis parallel to the flat side, an elliptical inner fluorine-doped depressed cladding $\left(\sim 22 \times 18 \mu \mathrm{m}^{2}\right.$, RI of 1.441), and a D-shaped undoped silica supercladding (RI of 1.444) The distance of the core layer from the flat surface of the Dfiber is of $\sim 13.5 \mu \mathrm{m}$. Note that the maximum transversal dimension of the D-fiber is exactly the same of the standard SMF: $125 \mu \mathrm{m}$. Evidently, the possibility to combine LPGs with Dshaped optical fibers have represented and still represent an open challenge for the scientific community. In this section, the scientific efforts already carried out in this field are resumed. The subject is treated as follows: first the attention is focused on the fabrication of LPGs into D-fibers, dividing the category in gratings involving physical modification of the core layer (intra-core LPGs) and gratings obtained by evanescent-wave mechanism; successively the different applications proposed for such structures are discussed. 

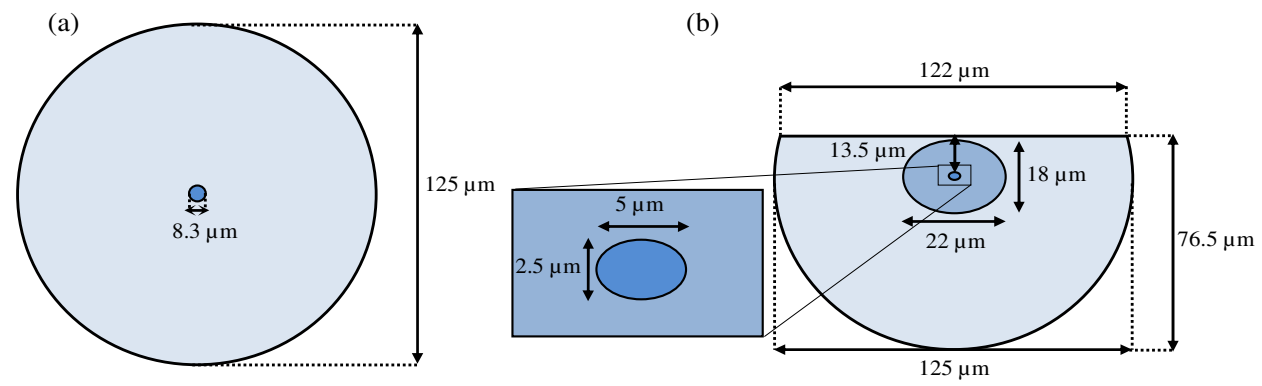

Fig. 1. Schematic diagram of the cross-section of (a) a standard SMF and (b) a D-fiber by KVH Industries, Inc. (not in scale).

\subsection{Intra-core LPGs}

Classically, a LPG is an axially periodic RI variation inscribed in the core of a photosensitive SMF by UV irradiation, which couples light from the fiber core into the cladding modes at discrete wavelengths. The index modulation produces a set of attenuation bands seen in the transmission spectrum of the optical fiber. The first paper presenting a LPG written in Dshaped SMF was dated 2004 (Allsop et al., 2004). Few data were given about the fiber structure: the D-fiber was originally designed for coupler fabrication. The core's radius was $4 \mu \mathrm{m}$, and the distance between its center and the flat of the " $\mathrm{D}$ " was $9 \mu \mathrm{m}$ with a cladding radius of $62.5 \mu \mathrm{m}$. The core was a composition of $\mathrm{GeO}_{2} / \mathrm{SiO}_{2}$ and the cladding was assumed to be $\mathrm{SiO}_{2}$. The D-fiber was not specifically designed to be photosensitive and so its photosensitivity was increased by hydrogenation at a pressure of 120 Bar for two weeks. The LPGs were fabricated using a frequency doubled argon ion laser at a wavelength of 244 $\mathrm{nm}$ with a point-by-point writing technique. Several grating periods were used from 140 to $400 \mu \mathrm{m}$ with a grating length of $5 \mathrm{~cm}$. Scrutinizing the transmission spectrum during fabrication, it was noticed that the attenuation bands grew in strength with a red shift but this strengthening and red shifting continued post-fabrication with shifts well in excess of $150 \mathrm{~nm}$ followed by a roughly comparable blue shift. An example of part of post-fabrication spectral evolution is shown in Fig. 2. The authors hypothesized this behaviour was due to different $\mathrm{H}_{2}$ diffusion rates from core and cladding.

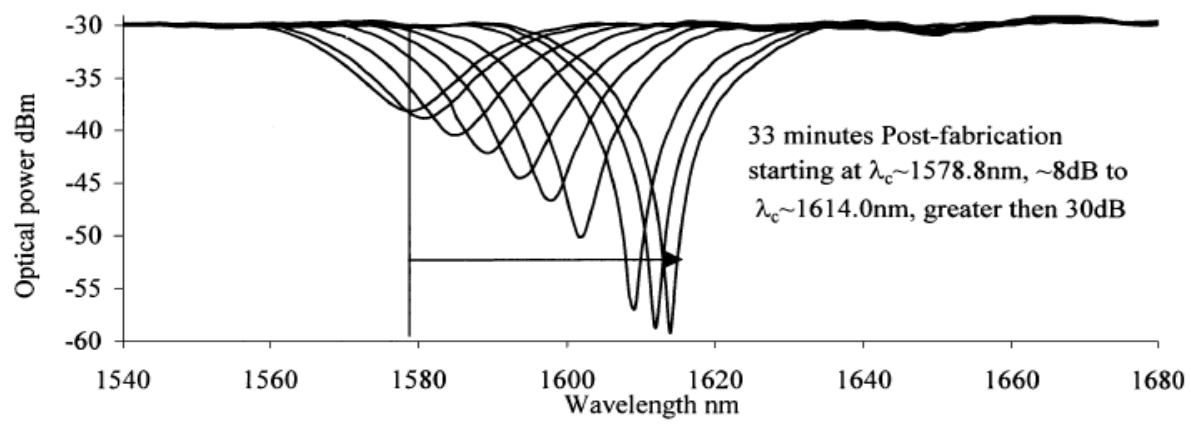

Fig. 2. Post-fabrication spectral evolution of an attenuation band from a D-fiber based LPG (period of $400 \mu \mathrm{m}$ ). Reproduced with permission from (Allsop et al., 2004). 
In the same year, LPGs were also fabricated within the D-shaped fiber supplied by KVH (Chen et al., 2004). To provide for comparison, LPGs were UV inscribed in both the Dfiber and standard SMF employing the point-by-point fabrication technique and a continuous-wave frequency-doubled Ar laser of $100 \mathrm{~mW}$ power. Prior to UV exposure, the fibers were photosensitized by a standard $\mathrm{H}_{2}$-loading treatment. Following inscription, the gratings were stabilized by thermal annealing at $80^{\circ} \mathrm{C}$ for 48 hours. Figs. $3(\mathrm{a})$ and (b) show typical spectra for two $4 \mathrm{~cm}$ long LPGs with periods of 490 and $380 \mu \mathrm{m}$ in standard SMF and D-fiber, respectively. In the D-fiber case, the birefringence results in the presence of two sets of broad loss peaks corresponding, respectively, to the two orthogonal polarization states.
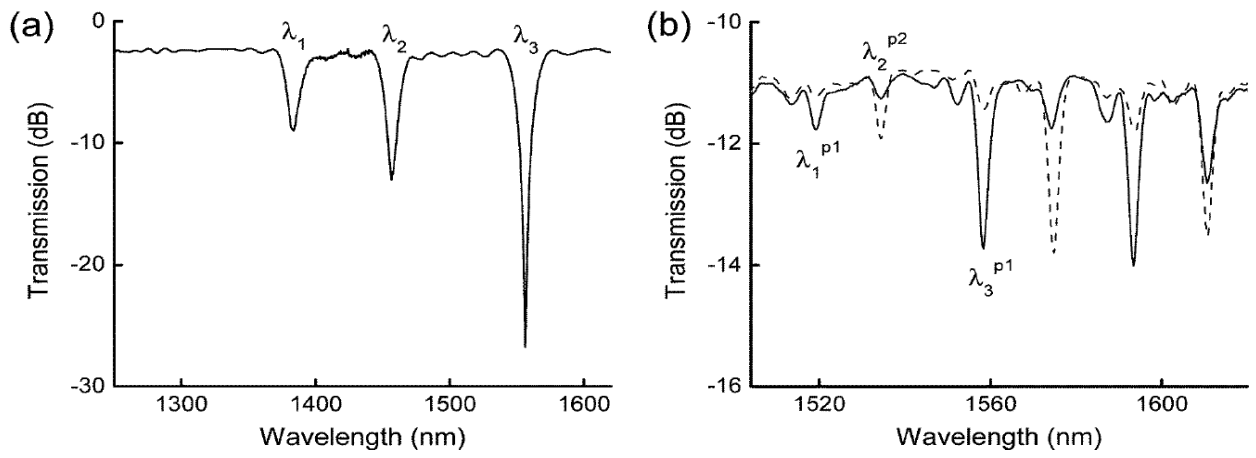

Fig. 3. Typical transmission spectra of LPGs in (a) standard SMF, and (b) D-fiber. There are two sets of resonances in D-fiber grating spectrum, corresponding to the two orthogonal polarization states, $\mathrm{P}_{1}$ and $\mathrm{P}_{2}$. Reproduced with permission from (Chen et al., 2004).

Obviously, D-fiber based LPGs can be also obtained by grating writing in standard SMF and successive side-polishing of the fiber section containing the grating (Tien et al., 2009a). In that case, a $2 \mathrm{~cm}$ long LPG with period of $380 \mu \mathrm{m}$ was written within a $\mathrm{H}_{2}$-loaded standard $\mathrm{SMF}$ using a $\mathrm{KrF}$ excimer laser with a wavelength of $248 \mathrm{~nm}$ and an amplitude mask. After grating writing, the fiber section containing the LPG was double-sided-polished: during the process, the polishing depth was monitored by checking the transmitted light power levels. Fig. 4 shows the original LPG transmission spectrum and that obtained after polishing: a red shift in the range of several nanometres is observable.

Finally, special attention has to be dedicated to the air-gap LPG (AG-LPG) first proposed in 2009 (Fu et al., 2009). Differently from standard UV written LPGs, in fact, here the grating is an axially periodic structural modification of the core layer (periodic AGs). The fabrication steps of such a D-fiber based LPG are resumed in Fig. 5. In particular, starting from a standard SMF, the first step is to side-polish the fiber to yield a flat polished surface on the cladding layer (see Fig. 5(a)). The distance between the core and the flat polished surface is $\sim 12-15 \mu \mathrm{m}$. The second step is to coat a negative photoresist with a thickness of $\sim 10 \mu \mathrm{m}$ on the flat polished surface of the fiber. After it is exposed and developed under UV light, the fiber is coated with a periodic $(410 \mu \mathrm{m})$ resist (see Fig. $5(\mathrm{~b}))$. Finally, the fiber is HF etched to yield a $3 \mathrm{~cm}$ long AG-LPG (see Fig. 5(c)). Note that, owing to the isotropic nature of the HF based etching, experimentally the sections of AGs appear to be laddershaped. 


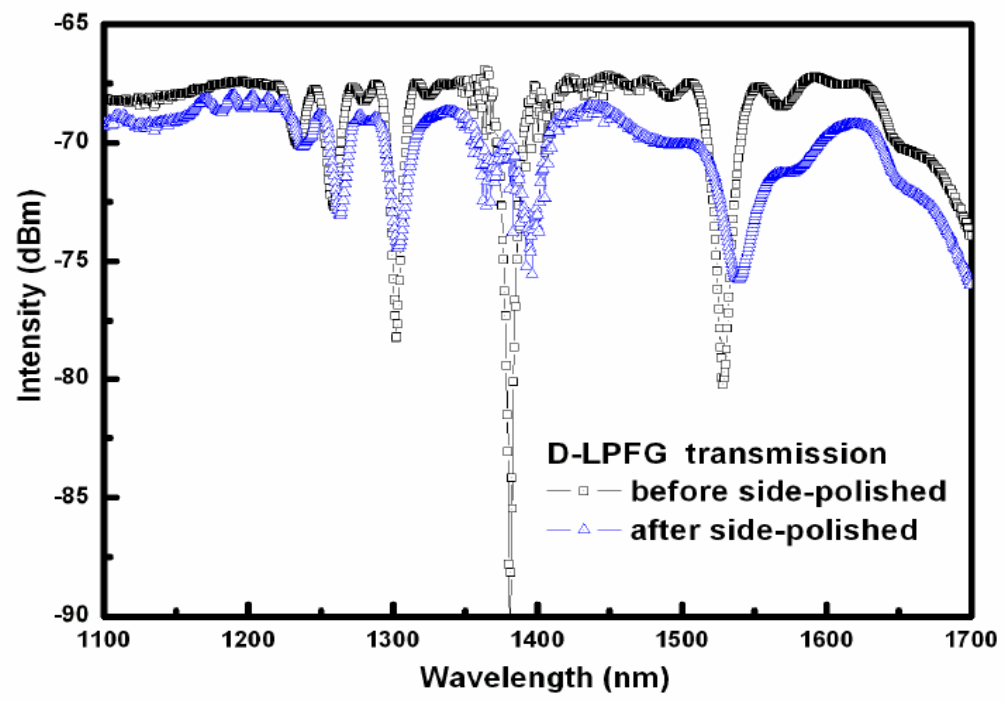

Fig. 4. Transmission spectra before and after side-polishing of the LPG. Reproduced with permission from (Tien et al., 2009a).

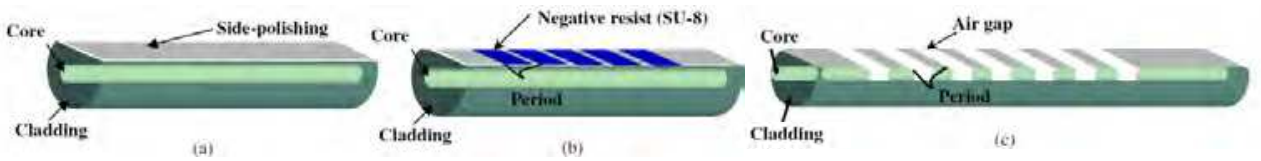

Fig. 5. Schematic diagram of the fabrication steps for the realization of AG-LPGs (not in scale). Reproduced with permission from (Fu et al., 2011).

\subsection{Evanescent-wave LPGs}

Along a LPG, the core periodic modification is substantially necessary to induce an effective RI modulation with the same periodicity on the core mode of the SMF: this is the real perturbation able to force the modal coupling between the core and cladding modes. On the other hand, the direct modification of the interested fiber layer is not the only way to force an effective RI modulation of the modes propagating within that layer. Uniform HRI nanocoatings, for example, have been proved to induce strong changes on the cladding modes effective RIs via evanescent-wave (Cusano et al., 2009b). By exploiting the same principle, HRI coatings should be able to induce changes on the core mode effective RI if the diameter of the cladding layer is opportunely reduced (Cusano et al., 2007). The first evanescent-wave D-fiber based LPG has been demonstrated in 2009 (Jang et al., 2009). The cladding layer of a SMF was substantially reduced using the side-polishing method to enhance the interaction between the core mode and the external medium via evanescent-wave. In particular, the unjacketed fiber was placed in a bent groove (curvature radius of $250 \mathrm{~mm}$ ) in a quartz block and was held by a UV epoxy. The block was polished until the cladding of the fiber was nearly removed. Successively, the LPG pattern was formed on the side-polished surface using a photolithography process: i) photoresist was spread on the polished surface by spin- 
coating (thickness of $2.1 \mu \mathrm{m}$ ) and ii) the LPG pattern was formed by UV exposure through a shadow long-period mask followed by a development process. The evanescent-wave LPG was $25 \mathrm{~mm}$ long, with a period of $600 \mu \mathrm{m}$. Fig. 6(a) and (b) show a schematic diagram of the LPG and a microscope image of a section of the LPG, respectively. In this case, the HRI photoresist increases the effective RI of the core mode along the coated regions, whereas it is left unperturbed elsewhere.

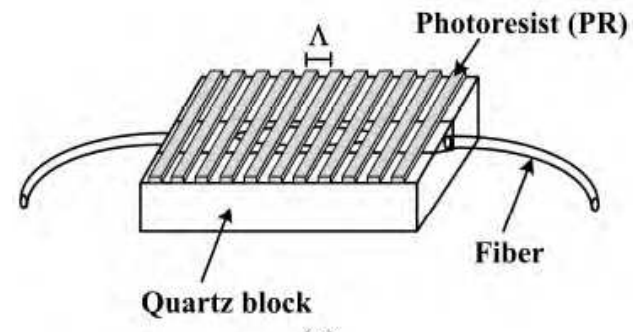

(a)

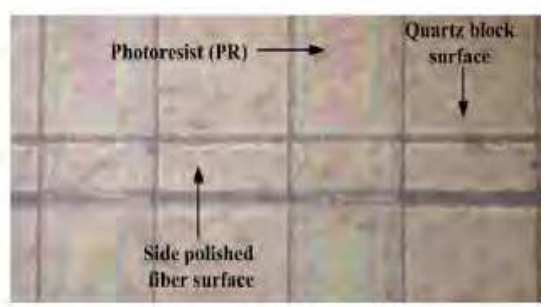

(b)

Fig. 6. Side-polished fiber with a LPG pattern: (a) schematic diagram (not in scale); (b) microscope image. Reproduced with permission from (Jang et al., 2009).

The same principle of operation has been exploited to develop an evanescent-wave LPG also along a D-shaped PCF (H. Kim et al., 2010). Figs. 7(a) and (b) schematically show the device evolution along the longitudinal direction of the fiber at different steps of its fabrication; Fig. 7(c), instead, shows the transversal section of one of the coated regions along the final device. The utilized PCF had pitch of $5.1 \mu \mathrm{m}$, air hole size of $1.3 \mu \mathrm{m}$, and core and cladding diameter of 10 and $130 \mu \mathrm{m}$, respectively. To fabricate the D-shaped PCF, two polishing processes were performed. In the first one, the PCF was placed on a Vgroove along a quartz block (curvature radius of $90 \mathrm{~cm}$ ) and fixed by using a UV-curable epoxy. The structure was ground down on a brass plate with $\mathrm{Al}_{2} \mathrm{O}_{3}$ powder. Then, the slurry on the flat surface was washed by ultrasonic cleaning with de-ionized water and successively dried at $100^{\circ} \mathrm{C}$ for $10 \mathrm{~min}$. The air holes on the polished surface were covered by using a UV-curable epoxy (RI of 1.56) in order to remove external materials infiltrated into air holes: the transmission loss caused by the epoxy was measured to be less than 0.2 $\mathrm{dB}$. For the second polishing process, the ground PCF was positioned on a polyurethane plate and polished with $\mathrm{CeO}_{2}$ powder to diminish the surface roughness of the D-shaped PCF. Also after this step, the polished surface was washed and dried. The measured residual cladding thickness was $\sim 0.1 \mu \mathrm{m}$. The previously described photolithography processes were used for the deposition of a uniform photoresist overlay and for its patterning. Two D-shaped PCF samples with different surface structures were fabricated: one presenting a uniform thin film of resist (thickness $\mathrm{d}$ of $3.5 \mu \mathrm{m}$ ) and the other one with the same resist layer patterned with a period of $400 \mu \mathrm{m}$ to produce the LPG. Figs. 7(d) and (e) show the transmission spectrum of the two D-shaped PCF samples. In both cases, dips associated to modal coupling are present. In particular, the PCF-based thin layer present two different dips at $\sim 800$ and $\sim 1320 \mathrm{~nm}$ : they are probably due to coupling of the core mode with overlay modes. On the other side, for the surface LPG these two dips are narrower and reduced in depth; in addition also a dip slightly beyond $1000 \mathrm{~nm}$ is present, probably the only one effectively associated to the grating. 
(a) Photoresist thin layer

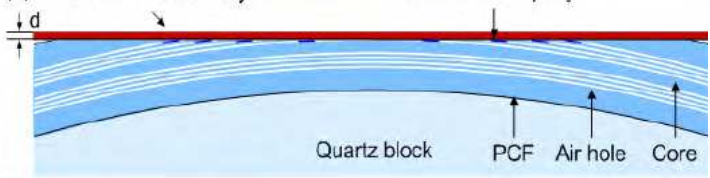

(b)

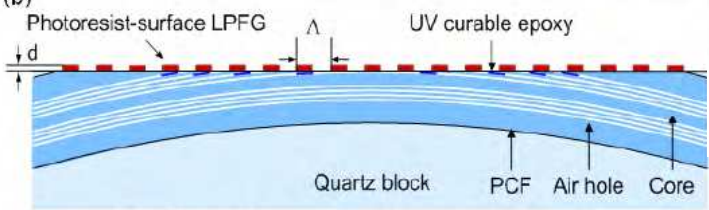

(c)

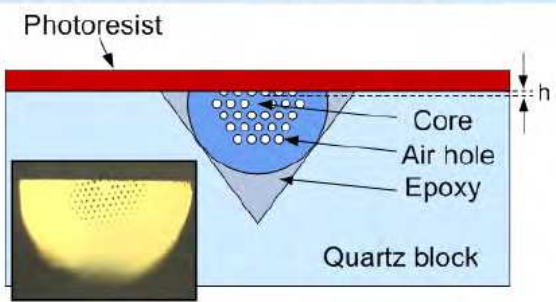

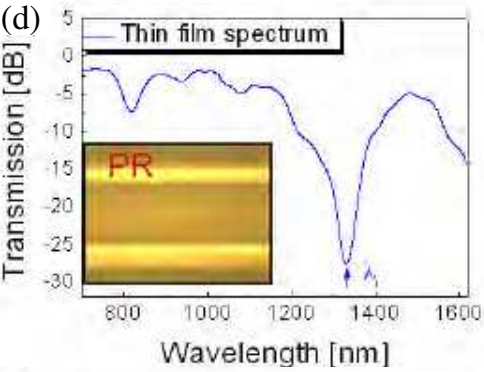

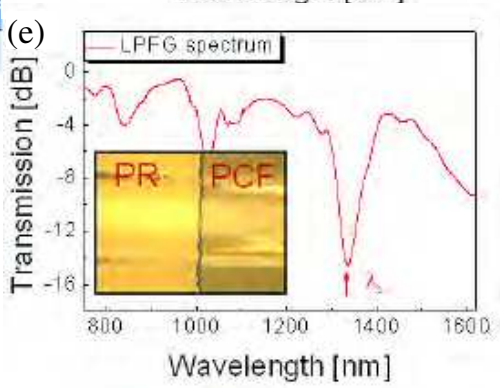

Fig. 7. Schematic diagram (not in scale) of (a) the uniform thin layer along the D-shaped PCF, (b) the PCF-based surface LPG, and (c) the cross section of the D-shaped PCF with the photoresist overlay; transmission spectrum of (d) the PCF-based thin layer and (e) the PCFbased surface LPG. Reproduced with permission from (H. Kim et al., 2010).

The idea at the basis of the previous evanescent-wave LPG configuration is very interesting, but its fabrication procedure shows certain limitations: i) the fiber device has to be integrated in a bulk material for side-polishing, loosing its typical compactness and ii) the adoptable overlay types are limited to photoresist. To overcome this limits, we recently proposed a different approach to realize evanescent-wave D-fiber based LPGs (Quero et al., 2011). First of all, the D-fiber supplied by KVH (see Fig. 1(b)) was adopted: it simply needs superficial etching in correspondence of the flat surface to allow evanescent-wave interaction of the core mode (Fig. 8(a)). It also provides, at this stage, the possibility to tailor the SRI sensitivity of the device by a proper choice of the etching depth. Successively, as proof of concept, a basic polymeric overlay of atactic polystyrene (PS) was uniformly deposited along the fiber by dip-coating technique (Fig. 8(b)). Finally, the overlay was properly confined in correspondence of the core layer on the flat surface of the fiber (Fig. 8(c)) and periodically patterned (Fig. 8(d)) by laser micromachining techniques. The main advantage of this approach relies on the flexibility: PS was used only for validation, several HRI material can be adopted depending on the specific application. During the HF based etching procedure, a particular point to be taken into account is the different etching rates of the three layer constituting the D-fiber structure. In particular, it is necessary to etch the fluorine-doped inner cladding to obtain evanescent-wave interaction of the core mode and such a layer etches $\sim 1.4$ times faster than the silica super-cladding. However, the etching depth can be controlled by monitoring the transmitted power: a $2.5 \mathrm{~cm}$ etched sample presenting $5 \%$ optical power losses was selected. The correspondent transmitted spectrum is reported in Fig. 8(e) (black curve). During the second step, a uniform PS overlay (RI of 
1.59) was deposited along the etched D-fiber by dip-coating (thickness of approximately $1.4 \mu \mathrm{m}$ ): the correspondent transmitted spectrum is shown in red in Fig. 8(e). Evidently, the HRI overlay induces further optical losses, but, above all, forces selective spectral features probably due to a coupling mechanism of the guided light with overlay modes. To avoid that the spectral features of the LPG to be realized would be compromised by such selective spectral features, an excimer laser micromachining system $(\mathrm{KrF}, \lambda=248 \mathrm{~nm})$ was used to confine the overlay on the flat surface of the fiber: a strip $2 \mathrm{~cm}$ long and $30 \mu \mathrm{m}$ wide was realized in correspondence of the core. As observable from the spectral response of the device after confinement (green curve in Fig. 8(e)), the selective spectral features were no more observable and the transmission spectrum results quite flat. Finally, the evanescentwave LPG was realized via laser micromachining by periodically patterning the PS strip with a period of $500 \mu \mathrm{m}$ ( $250 \mu \mathrm{m}$ alternatively coated and uncoated): as shown in Fig. 8(f), the LPG transmission spectrum present three different attenuation bands located at $\sim 1360$, $\sim 1440$, and $\sim 1530 \mathrm{~nm}$.
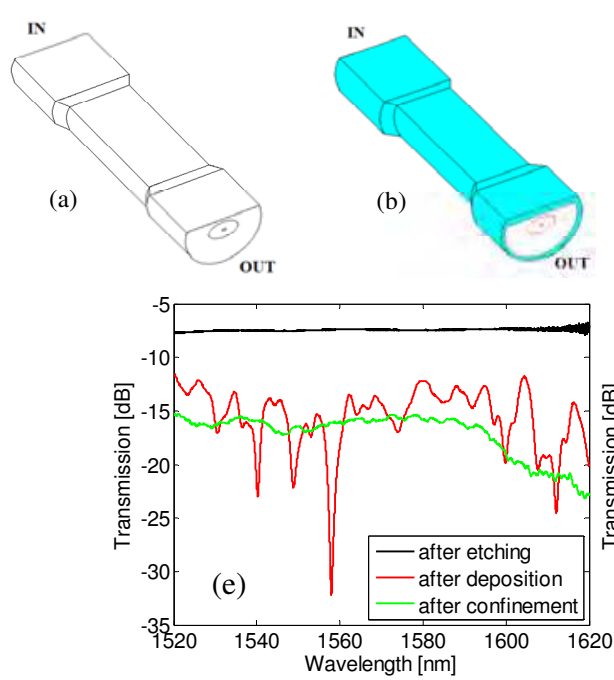
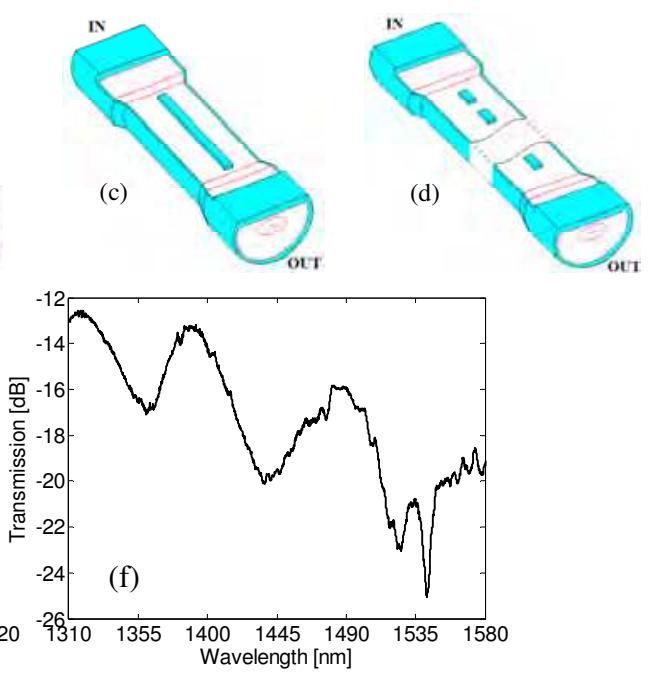

Fig. 8. Evanescent-wave LPFG: (a)-(d) Schematic diagram (not in scale) of the fiber structure step-by-step; transmission spectrum at (e) steps (a)-(c) and (f) step (d). (Quero et al., 2011).

\subsection{Applications}

D-fiber based LPGs have been demonstrated to be very useful devices, especially in the sensing field. In this section, the applications of such devices are briefly resumed: according to the previous sections, first intra-core and then evanescent-wave LPGs will be considered. The bending and orientational characteristics were the main aim of the first study about Dfiber based LPGs (Allsop et al., 2004). Typically increasing curvature causes splitting of LPG attenuation bands in standard SMF. Differently, the spectral evolution versus bending of one of the first D-fiber based LPGs is shown in Fig. 9. As observable, new bands appear under the influence of bending. Such transmission features are due to mode coupling coefficients that increase with curvature. The most sensitive bend-induced attenuation bands was characterized by a bend sensitivity of $12.55 \pm 2 \times 10^{-2} \mathrm{~nm} \cdot \mathrm{m}$, whereas the normal 
attenuation band had a sensitivity of $-1.735 \pm 8 \times 10^{-3} \mathrm{~nm} \cdot \mathrm{m}$. Also, it was found that the bend-induced bands were sensitive to the orientation of the bend with respect to the flat of the " $\mathrm{D}$ ", suggesting, in principle, a possible application as directional bend sensor for the Dfiber LPG. The grating was tested also versus temperature, revealing the possibility to use the LPG sensor also to discriminate between temperature and bending effects because it yields a reasonably well-conditioned sensitivity matrix.

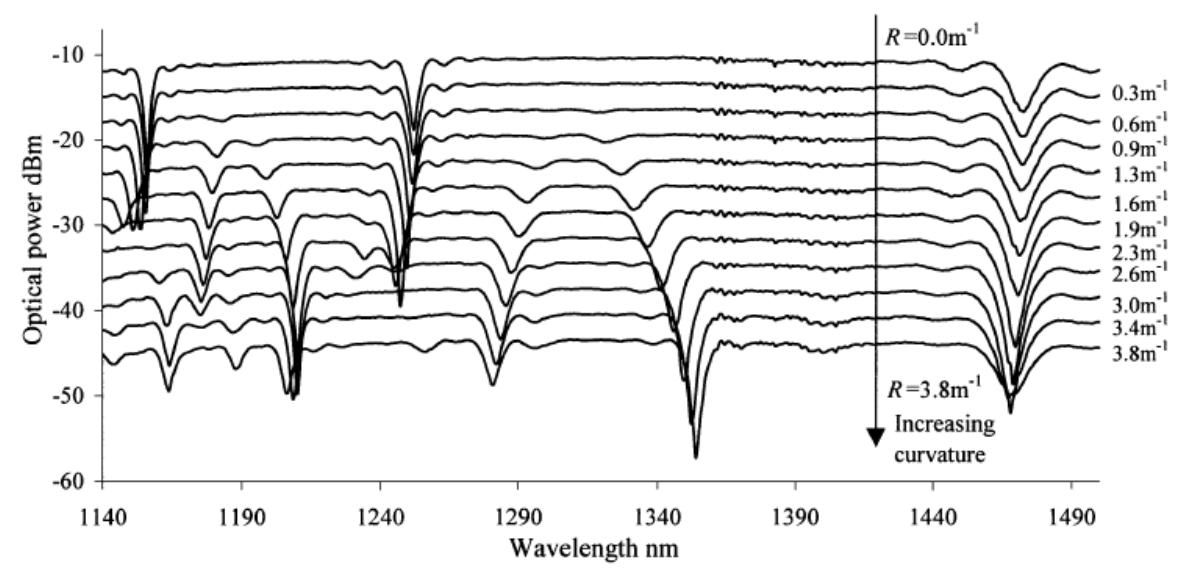

Fig. 9. Evolution of the transmission spectrum of a D-fiber based LPG (period of $380 \mu \mathrm{m}$ ) subjected to bending, showing the appearance of bend induced stop-bands. (Reproduced with permission from (Allsop et al., 2004)).

The application proposed for the first LPG inscribed within the D-fiber by KVH, instead, was as optical chemsensor with cladding etching to enhance sensitivity (Chen et al., 2004). A comparative investigation on the SRI sensitivity was conducted on LPGs in both D-fiber and the standard SMF by measuring the grating response to the aqueous sugar solutions, with sugar concentrations varying from $0 \%$ to $60 \%$. The LPGs presented in Fig. 3 were utilized and both of them were tested in standard configuration and after an etching time of $40 \mathrm{~min}$ in HF bath at $10 \%$ concentration (the etching rate was found to be the same for the two kinds of optical fiber). Evidently, the D-fiber device possessed an intrinsically higher SRI sensitivity and the etching significantly enhances the SRI sensitivity, especially for the Dfiber LPG. However, it is important to note that different grating periods were considered (shorter for the D-fiber) and this surely influenced the SRI sensitivity. The same authors also proposed a dual-parameter in-fiber sensor based on a hybrid LPG-FBG structure (Chen et al., 2005). The simultaneous measurement of temperature (FBG) and SRI (LPG) was proved. Always in 2004, the bending sensitivity characteristics of such D-fiber based LPGs were studied (D. Zhao et al., 2004a; D. Zhao et al., 2004b): their spectral response depends strongly not only on the curvature amplitude but also on the fiber orientation. Potential applications as directional shape sensor, bend-insensitive sensor, and two-axis curvature sensor (here a couple of LPGs is necessary) were hypothesized. Finally, the spectral characteristics of LPGs UV-written within the D-fiber supplied by KVH were also studied by Allsop et al. in 2006 (Allsop et al., 2006). The authors were able to fabricate LPGs with overlapping orthogonal polarization state attenuation bands: the use of such bands can 
considerably simplify the sensor interrogation. However, the spectral sensitivity of both orthogonal polarization states was measured with respect to temperature, rotation and bending. The temperature sensitivity was low compared to LPGs in standard SMF. Moreover, such LPGs devices produced blue and red shifts depending upon the orientation of the bend with measured maximum sensitivities of -3.56 and $6.51 \mathrm{~nm} \cdot \mathrm{m}$. The use of neighbouring bands to the overlapping orthogonally polarized attenuation bands to perform simultaneous measurement of temperature and bending was also demonstrated, which yielded a maximum polarization dependence curvature error of $\pm 0.08 \mathrm{~m}^{-1}$ and a temperature error of $\pm 0.05^{\circ} \mathrm{C}$. Since also the rotation of the bent LPG produced wavelength shifts, this type of LPG may be useful as a shape sensor and the polarization dependence can be reduced by using the overlapping orthogonal polarization state attenuation bands.

The effective scientific interest in D-fiber based LPGs is demonstrated also by the theoretical works regarding SRI measurements based on surface plasmon polariton (Tripathi et al., 2008; Tripathi et al., 2009), opening the way to the design of high performance chemical/biological sensors. The 2008 work studies the SRI sensing characteristics of metalcoated side-polished standard SMF gratings: both FBGs and LPGs. The authors used a simple approach for modelling side-polished SMF (Sharma et al., 1990) and demonstrated that the LPG-based sensor requires shorter grating lengths and higher metal thickness for a given sensitivity, making it more practical to realize. The 2009 work, instead, is focused on the SRI sensitivity characteristics of metal-coated LPGs operating in the power coupling regime corresponding to dual spectral resonance within D-fiber by KVH. The authors used a simple and sufficiently accurate first-order perturbation model (Kumar \& Varshney, 1984) and demonstrated that, by an optimum combination of metal thickness and core to flat surface separation, SRI sensitivity as high as $5971 \mathrm{~nm} /$ RIU (RIU - RI Unit) can be reached.

The double-sided polished D-fiber LPG proposed by Tien et al. in 2009 was first proposed as magnetic field sensor (Tien et al., 2009a). The magnetic sensing material was a Fe thin film with a thickness of $80 \mathrm{~nm}$, deposited by evaporation coating technique onto the doublesided-polished surface. The maximum blue shift experienced by the $1310 \mathrm{~nm}$ attenuation band (see Fig. 4) was $36 \mathrm{~nm}$ when the magnetic field was $153 \mathrm{kA} / \mathrm{m}$, corresponding to a sensitivity of about $0.24 \mathrm{~nm} /(\mathrm{kA} / \mathrm{m})$. The double-sided-polished D-fiber LPG was also proposed for liquid RI measurements in the bare configuration (Tien et al., 2009b), revealing a maximum SRI sensitivity of $143.396 \mathrm{~nm} / \mathrm{RIU}$.

To complete the intra-core LPGs category, the applications of AG-LPGs needs to be mentioned: first, they were tested for SRI measurements (Fu et al., 2009). Fig. 10(a) shows the transmission spectrum of a AG-LPG with period of $410 \mu \mathrm{m}$ for several SRIs (sugar solutions at different concentrations): differently from standard LPGs, a red shift as the SRI grows up is observable. In particular, in the 1.33-1.42 SRI range a linear behaviour has been pointed out for the attenuation band at $\sim 1550 \mathrm{~nm}$ with SRI sensitivity of $\sim 620 \mathrm{~nm} / \mathrm{RIU}$. This peculiar spectral feature can be explained by considering that the resonant wavelengths $\lambda_{\text {res,m }}$ of a LPG with period $\Lambda$ are determined by the following phase-matching condition (Vengsarkar et al., 1996; Shu et al., 2002):

$$
\lambda_{\text {res }, m}=\left(n_{\text {core }}^{\text {eff }}-n_{\text {cladding, } m}^{\text {eff }}\right) \cdot \Lambda
$$

where neff $_{\text {core }}$ and $n^{\text {eff }}$ cladding, $m$ are the effective RIs of the fundamental core mode and the mth cladding mode, respectively. For a standard LPG, SRI changes are able to modify only the cladding mode RIs: the higher is the SRI, the higher are the cladding mode RIs, leading to a 
blue shift of the attenuation bands. In the AG-LPG case, instead, SRI changes influence both the cladding and the core mode effective RIs. In particular, the SRI sensitivity is higher for the core mode and consequently a red shift as the SRI grows up is observable in Fig. 10(a). Successively, the AG-LPG has been proposed as humidity sensor (Fu et al., 2011). In this case, the polished surface of a $500 \mu \mathrm{m}$ period AG-LPG was coated with a calcium chloride $\left(\mathrm{CaCl}_{2}\right)$ thin film of $\sim 3 \mu \mathrm{m}$ thickness. Since $\mathrm{CaCl}_{2}$ is strongly hygroscopic, it is known as a drying agent or desiccant: in practice, the higher the relative humidity ( $\mathrm{RH}$ ) increases, the lower the RI of the $\mathrm{CaCl}_{2}$ thin film decreases. The device was tested in the $\mathrm{RH}$ range from $55 \%$ to $95 \%$ and a linear blue shift was observed for the grating attenuation band, revealing a sensitivity of about $1.36 \mathrm{~nm} / 1 \% \mathrm{RH}$. However, when the $\mathrm{RH}$ was increased from $85 \%$ to $95 \%$, no wavelength shift was observed because of the saturation of the chemical interaction between $\mathrm{CaCl}_{2}$ and $\mathrm{H}_{2} \mathrm{O}$ molecules. Finally, the thermal crosstalk was smaller than that of conventional LPGs with consequent less thermal compensation requirements.
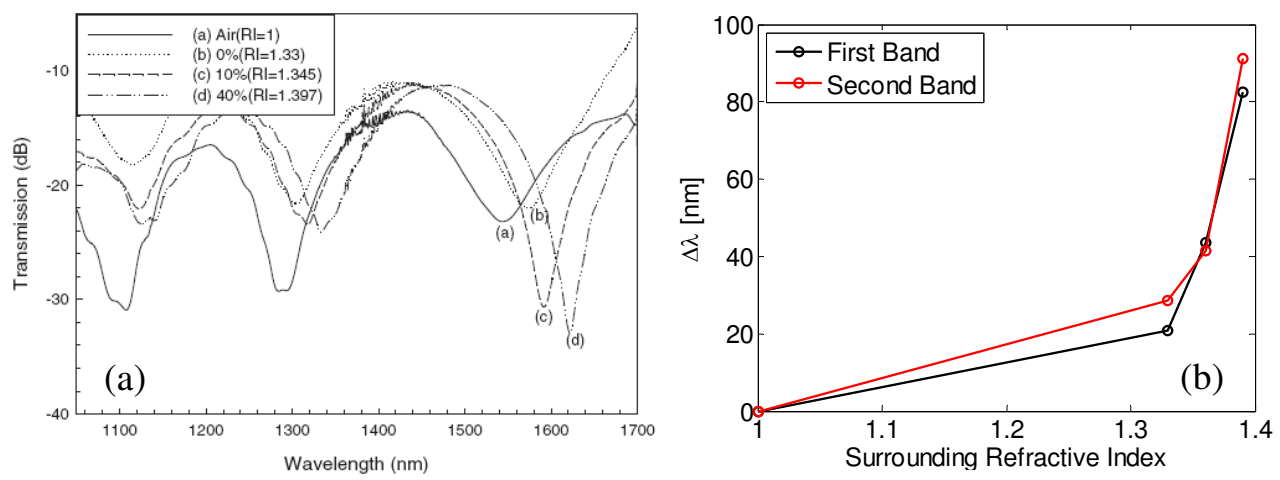

Fig. 10. (a) Transmission spectrum of an AG-LPG with period of $410 \mu \mathrm{m}$ for several SRIs. (Reproduced with permission from (Fu et al., 2009)). (b) Wavelength shift versus the SRI of the first two attenuation bands of the evanescent-wave LPG. (Quero et al., 2011)

As regards evanescent-wave D-fiber based LPGs, they represent a more recent research conquest. However, their spectral evolution versus the SRI is very similar to that of AGLPGs (see Fig. 10(a)): a red shift of the attenuation bands is observable for increasing SRIs. The first evanescent-wave LPG was proposed as sensitive DNA biosensor (Jang et al., 2009). The grating was used to detect the hybridization of single strand DNA (ssDNA). The wavelength shift were measured after the binding of the Poly-L-lysine, probe ssDNA and target ssDNA to the surface of the sensor. The overall shift induced by the DNA hybridization was $1.82 \mathrm{~nm}$ and the majority of it $(0.94 \mathrm{~nm})$ occurred in the first 9 min due to the rapid reaction with DNA hybridization. Recently, the same kind of evanescent-wave LPG - but PCF-based - has been proposed for SRI and temperature measurements demonstrating higher sensitivities as compared with conventional LPGs (H. Kim et al., 2011). As regards the more flexible evanescent-wave D-fiber based LPG recently proposed by us (Quero et al., 2011), it was characterized versus the SRI: Fig. 10(b) shows the wavelength shift of the attenuation bands located at $\sim 1360$ and $\sim 1440 \mathrm{~nm}$ (see Fig. 8(f)). Without optimization of the device parameters, SRI sensitivity around the water RI of $\sim 700$ and $\sim 625 \mathrm{~nm} / \mathrm{RIU}$ for the first and the second dip, respectively, has been pointed out. As 
consequence, this configuration represents an extremely attractive technological platform for chemical/biological sensing. In addition, the possibility to use HRI materials with different natures (electro-optical, magneto-optical, etc.) to fabricate such devices would open the way to self-functionalized evanescent-wave LPGs suitable for specific applications.

\section{Long period gratings in photonic crystal fibers}

Photonic Crystal Fibers, thanks to the new ways provided to control and guide light, not obtainable with conventional optical fibers, are driving an exciting and irrepressible research activity all over the World, starting in the telecommunication field and then touching metrology, spectroscopy, microscopy, astronomy, micromachining, biology and sensing. A PCF consists of regularly spaced air holes along the fiber cladding (Russell, 2003). The core of the PCF is formed by the introduction of a defect or a missed hole at the center of the fiber. According to the distinct mechanisms of light propagation in the core region, as shown in Fig. 11, PCFs fall into two general categories: (1) microstructure fiber or holey fiber in which the light is trapped by total internal reflection (TIR) in a solid core, which has a larger refractive index than the cladding region (i.e. index guided IG-PCF - see Fig. 11(a)), and (2) photonic bandgap fiber (PBG) in which the core of the fiber is hollow, and the light is trapped in the central lower-index region by a two-dimensional photonic bandgap created by the periodic cladding (i.e. hollow core HC-PCF - see Fig. 11(b)) (Frazao et al., 2008).
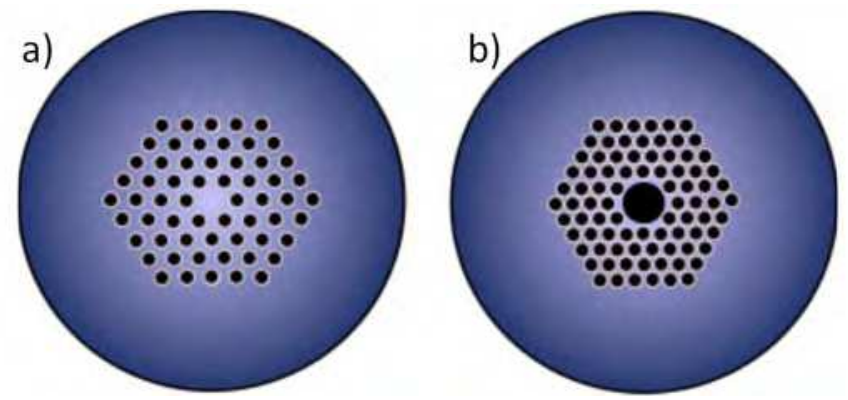

Fig. 11. Two main classes of photonic crystal fibers (PCF): index-guiding PBF (a) and photonic bandgap PBF (b). Reprinted with permission from Frazao, O., Santos, J.L., Araujo, F. M., \& Ferreira, L.A., (2008). Optical sensing with photonic crystal fibers. Laser \& Photonics Reviews, Vol. 2, No. 6, November 2008, pp.(449-459), ISSN 1863-8899.

In contrast to conventional fibers, most of PCFs are made by use of just a single material, typically fused silica, using the stack and draw technique, and their dispersion, mode-field confinement, single-mode range, and polarization dependence can be greatly controlled by size, shape, and pitch of the air holes. The structure of the PCF enables to have different types of fibers such as endless single mode, double clad, germanium or rare earth doped, high birefringence, and many others with peculiar features due to its manufacturing flexibility. This variety of choices permits the use of PCF in numerous applications spanning from communication components to sensors which measure physical parameters (temperature, pressure, force, etc.), chemical compounds in gas and liquids, and even biosensors. In particular, since 1999 (Eggleton et al. 1999, Espindola et a., 1999, Diez et al., 2000, Kakarantzas et al., 2002, Lim et al., 2004), the writing of LPGs into different types of 
PCFs with or without photosensitivity by the use of advanced laser processing techniques along with mechanical and chemical methods was quickly embraced expecting many new applications as sensors (Frazao et al., 2008) and fiber components (Y. Wang et al., 2007). For example, the resonant wavelength of an LPG written in a PCF is blue-shifted with the increase of grating periodicity, contrary to the usual case in a conventional single mode fiber (SMF). The next sections will present an overview of the long-period-grating written in IG$\mathrm{PCF}$ and in HC-PCF.

\subsection{LPGs in IG-PCFs}

The first photochemical grating written in PCF was achieved in 1999 (Eggleton et al. 1999). In this work a special PCF with a photosensitive Ge-doped core was used and the induced refractive index changes originated from the linear absorption of $\mathrm{Ge}$ oxygen-deficient centers with the maximum at $242 \mathrm{~nm}$ (common single-quantum inscription mechanism). UV-laser exposure is a common technique for writing gratings in Ge-doped fiber, but, however, typical PCFs have no photosensitivity because they are composed of pure silica, which is fully transparent in the UV spectral region. Therefore new non photochemical inscription techniques to fabricate LPGs in PCFs have been explored, that modify the refractive index in the fiber cladding either by heating (using $\mathrm{CO}_{2}$ laser light or an electric arc discharge) or by applying mechanical pressure. Kakarantzas et al. reported the first example of structural LPGs written in pure-silica solid core PCFs (Kakarantzas et al., 2002). As shown in Fig. 12, the gratings are realized by periodic collapse of air holes in the PCF via heat treatment with a $\mathrm{CO}_{2}$ laser. The resulting periodic hole-size perturbation produces core-to cladding- mode conversion, thus creating LPG in the PCF. In contrast with the LPGs written by UV light, which become unstable over time, $\mathrm{CO}_{2}$ laser-induced LPGs are temperature insensitive because of their structural perturbation along the fiber. This property can be utilized to obtain temperature-insensitive PCF-based devices, as demonstrated in (C. Zhao et al., 2008). The $\mathrm{CO}_{2}$ laser irradiation is a flexible, highly efficient, point-by-point, low cost technique for writing very compact (few $\mathrm{mm}$ ), deep notched $(>20 \mathrm{~dB})$ LPGs in a pure-silica PCFs without photosensitivity and the writing process can be computer-programmed to produce complicated grating profiles.

Similar to the $\mathrm{CO}_{2}$ laser is the arc induced technique. The LPGs are imprinted in PCFs with the electric arc discharge of a fusion splicer by using a point-by-point technique, which is extremely low cost since it eliminates the need for expensive laser systems or the need for pre-hydrogenation of the fiber (in comparison with UV gratings) and consequent postthermal annealing to stabilize the gratings (Humbert et al., 2003, Dobb et al., 2006). Repeatability was assured by always maintaining a constant arc current, arc duration and fiber tension. However, the reported number of the grating periods that is needed to achieve a comparable attenuation band is much larger than that of $\mathrm{CO}_{2}$ laser irradiation technique (Ju \& Jin, 2010). Practically all the LPGs written in PCFs by local heating rely on glass structure change and fiber deformation. Because of the high fictive temperature of pure silica, a high heating temperature (achieved with intense $\mathrm{CO}_{2}$-laser radiation or an electric arc) is needed to cause significant glass structure change. Thus, one of the serious disadvantages of these methods is the collapsing of fragile PCF holes, especially in the case of PCFs with relatively large holes, which results in a high insertion loss. Another disadvantage originates from the irregularity of period deformation. A recent study shows that by applying tension to the fiber during the writing process, through the mechanism of 
frozen-in viscoelasticity, it is possible to write strong gratings in PCFs with a dosage of $\mathrm{CO}_{2}$ laser radiation low enough not to cause any signification fiber structure deformation $(\mathrm{H}$. Lee \& Chiang, 2009).

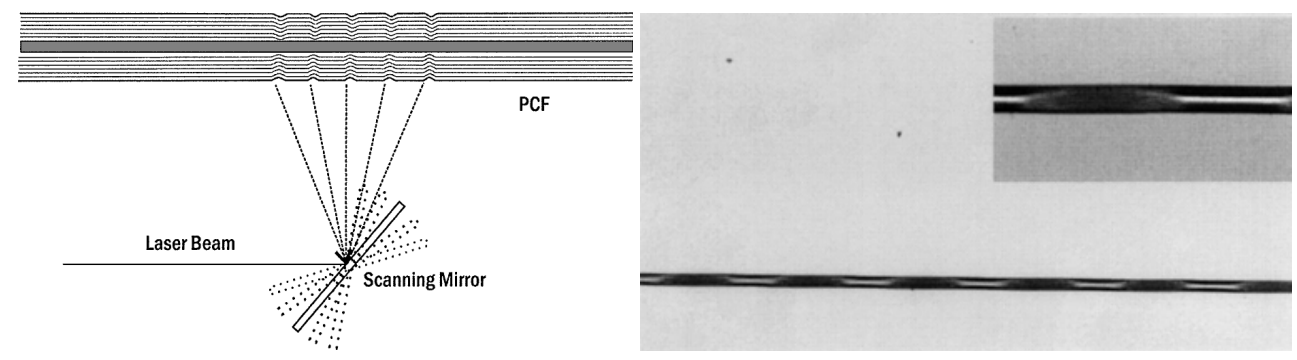

Fig. 12. Fabrication of a LPG based on microcollapsing the PCF holes with a CO2 laser beam (left) and optical micrographs of a section of the LPG after the holes at the parts heated by the $\mathrm{CO}_{2}$ laser have completely collapsed (right). The inset shows a magnification of one period. Reprinted from (Kakarantzas et al., 2002) with permission of Optical Society of America.

More recently the fabrication of LPG in PCFS was successfully demonstrated by use of a high-intensity femtosecond laser via a multiphoton absorption process. In order to avoid some drawbacks of the femtosecond laser technique, such as low efficiency (Allsop et al., 2008) or a $\mathrm{H}_{2}$-loading pre-procedure (Fotiadi e al., 2007), S. Liu et al. propose to tightly focus femtosecond infrared beam onto the holey inner-cladding region of a PCF (Liu et al., 2010). As shown in Fig. 13, the high intensity femtosecond-laser irradiation results in the filling of air-holes through possibly a laser-induced micro-explosion and redeposition process, which modifies the waveguide structure and forms an LPG. Fig. 13 shows the evolution of transmission spectrum with an increasing number of grating periods. Two resonant dips (A: $1540 \mathrm{~nm}, 26 \mathrm{~dB}$; B: $1370 \mathrm{~nm}, 5 \mathrm{~dB}$ ) were observed for the LPG with 13 periods and the insertion loss is $2 \mathrm{~dB}$. Furthermore, several LPGs were fabricated with similar inscription parameters but with pitch varying from 340 to $390 \mu \mathrm{m}$. Fig. 13 shows the measured relation between the resonant wavelength (the resonant dip A) and the grating pitch.

Mechanical pressure has provided another direct and flexible means for LPG inscription in PCF (Lim et al., 2004, D. Lee et al., 2006, Parka et al., 2006). Pressure on the fiber surface with a periodic grooved plate induces periodic index changes in the fiber. With this method the strength and the resonant wavelength of the mechanically induced LPG can be easily tuned simply by adjusting the grating period and the pressure applied on the PCF. The efficiency of the mode coupling between the core mode and a cladding mode varies with pressure. LPGs with proper lengths and periods have to be selected such that mode coupling occurs at the predetermined wavelength. However, the coupling was found to be highly polarizationdependent and dependent on the angular position where stress was applied (D. Lee et al., 2006). The highly polarization-dependent broadband coupling was observed due to the unique beat-length dispersion between the core-mode and cladding-mode, which could find potential applications in wide-band polarization dependent loss (PDL) compensation. Acousto-optical interaction can also cause mode coupling between different modes if phase matching conditions are satisfied. The acoustic LPG has great advantages in terms of tuning 
range and speed. Therefore, it has been extensively studied and is enabling numerous practical applications in optical communication. The acoustic LPG built on a PCF can make a function with the tuning range over $1000 \mathrm{~nm}$ as a single optical element (Hong et al., 2008). In conclusion, as synthetically illustrated, the integration of LPGs in PCFs can be achieved with different, complementary techniques. This provides new promising platforms for developing novel devices, for application in telecommunication and in sensing, combining the unique properties of the PCFs with the peculiarities of the PLGs.
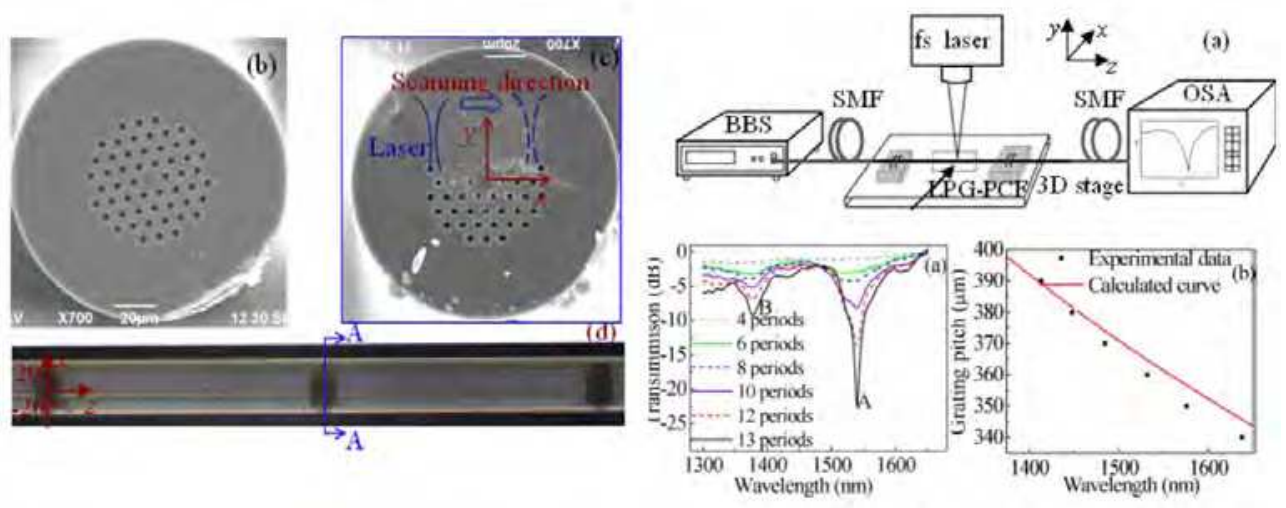

Fig. 13. (Top right a) Experimental setup for LPG fabrication. Scanning electron micrographs of LMA-10 PCF cross section (left b) before and (left c) after femtosecond-laser treatment. (left d) Top view of femtosecond-laser fabricated LPG. (Bottom Right a) Evolution of the transmission spectrum of LPG with increasing number of grating periods. (Bottom Right $b$ ) Relation between grating pitch and resonant wavelength. Reprinted with permission from Liu, S., Jin, L., Jin, W., Wang, Y., \& Wang, D. N. (2010). Fabrication of Long-Period Gratings by Femtosecond Laser-Induced Filling of Air-Holes in Photonic Crystal Fibers. IEEE Photonics Technology Letters, Vol. 22, No. 22, November 2010, pp. (1635-1637), ISSN 10411135 (c) 2010 IEEE.

\subsection{LPGs in HC-PCFs}

As discussed above, a large number of gratings have been demonstrated in different types of IG-PCFs by the use of various fabrication techniques while the development of efficient techniques useful to write LPG structure in HC-PCFs (Smith, 2003; Frazao et al., 2008) is a hard challenge for research community. Since more than $95 \%$ of the light propagates in the core-air of an HC-PCF and not in the glass (see Fig. 11(b)), such fibers offer a number of unique features including lower Rayleigh scattering, reduced nonlinearity, novel dispersion characteristics, and potentially lower loss compared to conventional optical fibers (Smith et al., 2003; West et al., 2004). In addition, the hollow core characteristic also enables enhanced light/material interaction, thus providing a valuable technological platform for ultrasensitive and distributed biochemical sensors. However, periodic index modulations usually required to realize mode coupling in LPG devices are not easy to achieve in HC-PCFs (Ozcana \& Demircib, 2004; Frazao et al., 2008). The main issue relies on the difficulty in introducing UV-induced refractive index modulation since up to $95 \%$ of the light energy is confined within the air-core. Additionally, the alternative use of localized fiber tapers could 
induces holes collapsing in the cladding region preventing the low loss propagation of light in the hollow core.

To bypass these technological difficulties and at same time to keep the concept of the PBG fiber, few years ago some LPG structures were demonstrated in a new kind of bandgapguiding fibers such as fluid-filled PBG fibers. Such fiber was composed of two different materials and can be fabricated by taking a solid core PCF with air-holes in the cladding and filling the holes with a high index fluid (Kuhlmey, 2009). The first LPG in fluid-filled PBG fibers was formed by inducing periodic mechanical stresses on the fiber in 2006 (Steinvurzel, 2006a, 2006b). The periodic stress-induced deformations of the fiber force light coupling between core mode and higher order modes. On the same kind of fiber also an electric-arc induced LPG was demonstrated (Iredale, 2006). One year later, it was proposed a rewritable self-assembled LPG in air-core PBG fibers (Ozcana \& Demircib, 2007). The LPGs were written by filling the air-core region of the fiber with a solution containing polystyrene microspheres. The microspheres are self-assembled into a periodic structure as the liquid inside the fiber evaporates, forming the long-period grating.

In 2008 instead, Wang et al. demonstrated the first example (as authors declared) of gratings written in an HC-PCF (Y. Wang et al, 2008). They proposed the use of a focused $\mathrm{CO}_{2}$ laser beam to periodically deform/perturb air holes along the fiber axis (used HC-PCF: CrystalFiber HC-1500-02). Figs 14(a) and 14(b) compare the cross-section of the unperturbed and $\mathrm{CO}_{2}$ treated fiber. The focused $\mathrm{CO}_{2}$ beam scans periodically the HC-PCF causing the ablation of glass on the fiber surface and the partial or complete collapse of air holes in the cladding. The outer rings of air holes in the cladding, facing to the $\mathrm{CO}_{2}$ laser irradiation, were largely deformed; however, little or no deformation were observed in the innermost ring of air holes and in the air core. As a result, periodic index modulations are achieved along the fiber axis due to the periodic perturbation (see Fig. 14(c)). Compared with the fabrication parameters for writing a grating in a solid-core PCF (Ju et al. 2004) a lower average laser power and shorter total time of laser irradiation are typically used to write a LPG in an HC-PCF. A proper choice of the fabrication parameters is critical for the fabrication of such LPG. High energy pulses with a long irradiation time may cause large deformation or collapsing of the holes and thus a higher insertion loss, while low energy pulses with short irradiation time may be insufficient to inscribe an LPG. Fig. 14(d) shows the measured transmitted spectrum of a 40-period LPG. It was retrieved the 3dB-bandwidth is about $5.6 \mathrm{~nm}$, which is much narrower than that of the LPGs with same number of grating periods in conventional SMFs (Bhatia et al., 1999) and in IG-PCFs (Morishita \& Miyake, 2004). Besides, Wang et al. believe that for the LPG written in HC-PCF, periodic perturbations of the waveguide (geometric) structure could be the dominant factor that causes resonant mode coupling, although the stress relaxation-induced index variation may also contribute a little (Y. Wang et al., 2008, 2010). Recently, the same group theoretically investigated the LPGs fabricated in HC-PCFs with a pulsed $\mathrm{CO}_{2}$ laser (Jin et al., 2011). By the use of the coupled local-mode theory, they numerically modelled the transmission and the polarization properties of the LPGs. They found that resonant couplings are resulted from the periodic modification of the fundamental and the higher order mode fields. As a result, two highly polarization dependent resonant dips are observed. Finally, they also investigated the spectral response versus the grating pitch (see Fig. 14(e)). Accordingly with LPG in IG-PCFs (Morishita \& Miyake, 2004; Petrovic, 2008) and in disagreement with LPG in SMFs (Vengsarkar, 1996), the LPGs written in the HC-PCFs have negative relationship between resonant wavelength and grating pitch. 

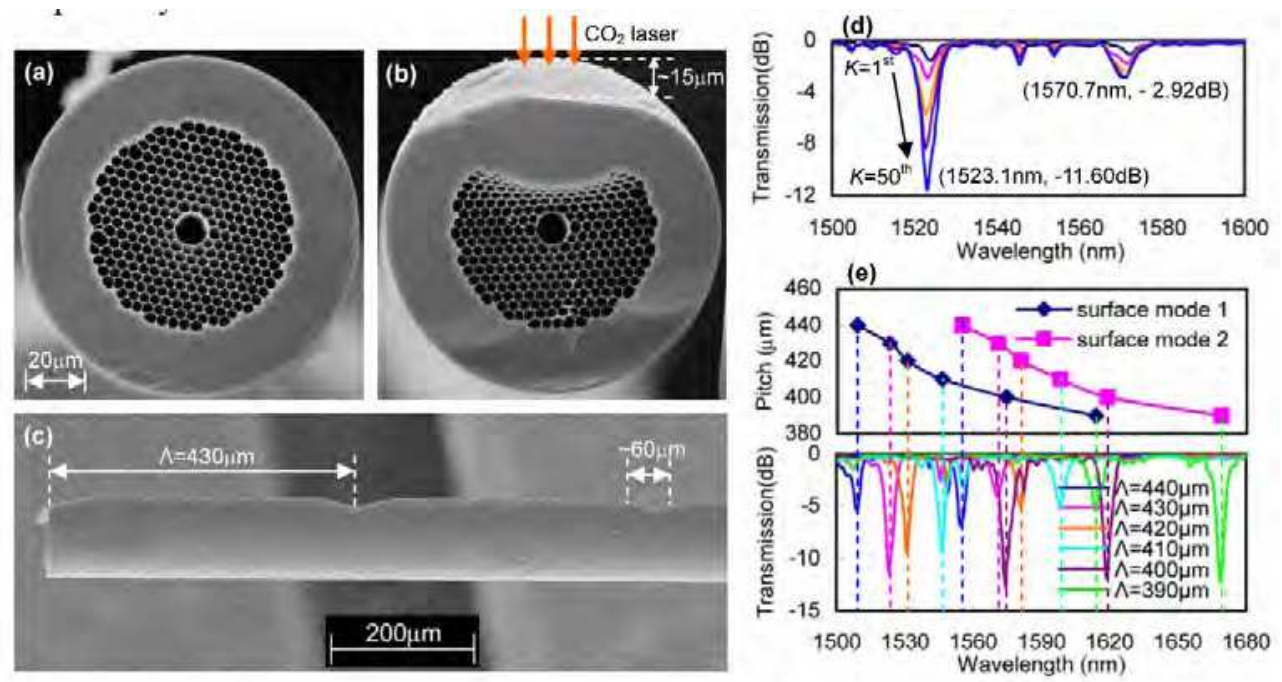

Fig. 14. Scanning electron micrographs of HC-PCF cross-sections (a) before and (b) after $\mathrm{CO}_{2}$ laser irradiation; (c) LPG on HC-PCF with 50 scanning cycles; (d) Evolution of the transmitted spectrum of LPG with 40 periods and a grating pitch of $430 \mu \mathrm{m}$ with increasing number of scanning cycles; (e) Variation of LPFG resonant wavelengths with grating pitch. Reprinted from (Y. Wang et al., 2008) with permission of Optical Society of America.

Recently, the authors of the present work have investigated the possibility to use a modified Electric Arc Discharge (EAD) technique (such as a pressure assisted EAD technique) to fabricate LPGs in HC-PCFs (Iadicicco et al, 2011a, 2011b). The fabrication procedure relies on the combined use of EAD step, to locally heat the HC fiber, and of a static pressure slightly higher than the external one inside the fiber holes, to modify the holes. This procedure permits to preserve the holey structure of the host fiber avoiding any hole collapsing and it enables a local effective refractive index change due to the size and shape modifications of core and cladding holes. EAD procedure has been carried out by a commercial fusion splicer unit (Sumitomo Type-39). To achieve an arc-discharge that would locally heat the fiber (avoiding any permanent distortion) fusion current and arc duration were manually selected to approximately $13 \mathrm{~mA}$ and $300 \mathrm{~ms}$, respectively. Besides, to force a static pressure inside the fiber holes, one end of the hollow-core fiber was EAD treated in order to force hole collapsing in both core and cladding region while the other end was connected to the needle of a $1 \mathrm{ml}$ syringe. Before any EAD step, static pressure inside core and cladding holes was imposed by decreasing the syringe volume of $20 \%$. A microscope image of the HC fiber cross-section before and after the pressure assisted EAD procedure is show in Figs. 15(a) and 15(b), respectively. By image analysis it is possible to retrieve that the external diameter of the fiber is reduced from $120 \pm 1 \mu \mathrm{m}$ to $117 \pm 1 \mu \mathrm{m}$ as well as the inner diameter of the external solid silica region from approximately $70 \pm 1 \mu \mathrm{m}$ to $65 \pm 1 \mu \mathrm{m}$. Moreover, the core size resulted enlarged passing from $11 \pm 1 \mu \mathrm{m}$ to $13 \pm 1 \mu \mathrm{m}$ whereas none air-hole rings was found collapsed even if changes in shape and size are evident. It is important to remark that further optimization margins exist by controlling the EAD procedure through fusion current, arc duration and syringe volume decreasing while the 
parameters used for first prototyping allowed to preserve the fiber band-gap since negligible insertion losses were observed. Finally, the LPG writing was possible by spatially repeating the EAD procedure along the host fiber by the use of a micro-controlled translation stage. Fig. 15(c) shows the side view of an LPG with 20 periods and pitch of $400 \mu \mathrm{m}$. In comparison with grating notches provided by $\mathrm{CO}_{2}$ laser approach in Fig. 14, the pressure assisted EAD procedure permits to avoid the strong impairment of final device and reduce the polarization dependent due to the asymmetric perturbations.

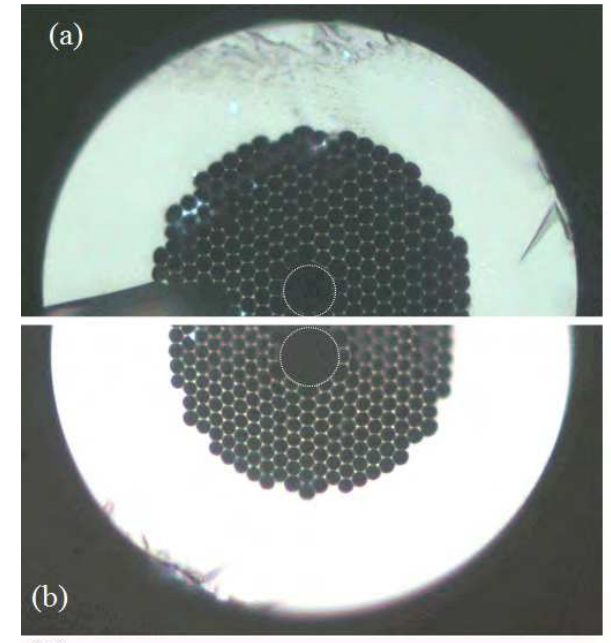

(c)

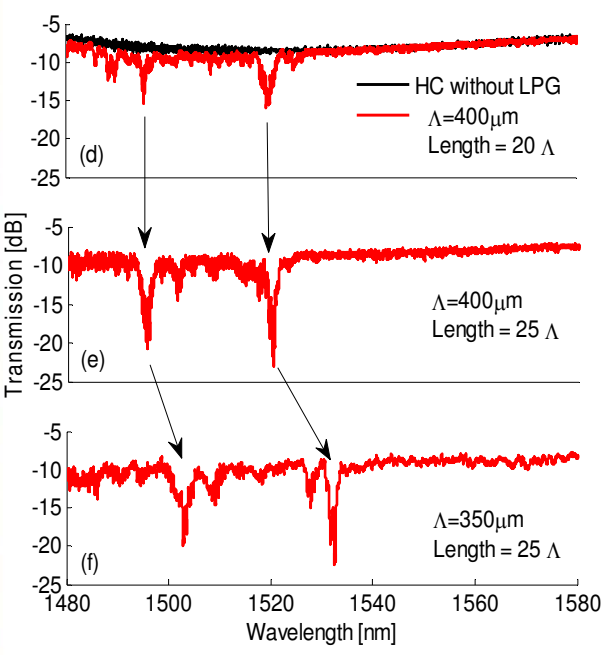

Fig. 15. Optical microscopy images of HC-PCF cross-section (a) before and (b) after EAD procedure; (c) Optical microscopy image of LPG with $\Lambda=400 \mu \mathrm{m}$ (20 periods); transmitted spectra of LPG in HC-PCF with (d) 20 periods and $\Lambda=400 \mu \mathrm{m}$, (e) 25 periods and $\Lambda=400 \mu \mathrm{m}$, and (f) 25 periods and $\Lambda=350 \mu \mathrm{m}$. (Iadicicco et al., 2011).

Fig. 15(d) plots the transmitted spectrum of the LPG realized with 20 periods and a pitch of $400 \mu \mathrm{m}$. Despite the limited grating length (approx. 8mm), the transmitted spectrum clearly exhibits attenuation bands due to resonant coupling of the fundamental core mode to leaky higher order modes (core or surface-like). Resonant wavelengths are approximately at $1495.3 \mathrm{~nm}$ and $1519.8 \mathrm{~nm}$ with attenuation depth of about $5 \mathrm{~dB}$ and $6 \mathrm{~dB}$ respectively. The $3 \mathrm{~dB}$ bandwidth for both resonances was found to be $1.2 \mathrm{~nm}$ and $2.0 \mathrm{~nm}$, respectively, and thus narrower than the common LPGs written in SMFs (Bhatia ,1999). Besides the geometric variation, it is worth highlighting that EAD also induces silica refractive index change due to stress relaxation induced by local hot spots. However, accordingly with the LPG based on $\mathrm{CO}_{2}$ laser we believe that the effective refractive index modulation is principally related to geometrical perturbation since the confinement of the fundamental mode $(>95 \%)$ within air core. Moreover, from Fig. 15(d), it is also possible to observe background oscillations or ripples (in spectra with or without LPG) attributable to different effects: i) Fabry-Perot effect due to HCF-SMF splicing ii) accordingly to [13], HOM (higher order modes) weakly excited in the HC fiber. Besides, Fig. 15(e) plots the spectrum of a LPG with 25 periods and $\Lambda=$ 
$400 \mu \mathrm{m}$. As expected, two attenuation bands at $1495.4 \mathrm{~nm}$ and $1520.3 \mathrm{~nm}$ (very close to attenuation bands shown in Fig. 15(d) ) with depth of $9 \mathrm{~dB}$ and $12 \mathrm{~dB}$ and bandwidth of $1.4 \mathrm{~nm}$ and $1.5 \mathrm{~nm}$, respectively, can be observed. Fig. 15(f), instead, plots the transmitted spectrum of a LPG with 25 periods and $\Lambda=350 \mu \mathrm{m}$. It seems that the resonant wavelengths increase with the decrease in the grating pitch, which is opposite to the behavior of LPFGs written in the conventional SMFs (Bhatia, 1999), but agrees with the above presented results (Y. Wang, 2008; Morishita \& Miyake, 2004). Here, two attenuation bands at $1503.0 \mathrm{~nm}$ and $1532.2 \mathrm{~nm}$ with depth of $9 \mathrm{~dB}$ and $11 \mathrm{~dB}$ and bandwidth of $0.9 \mathrm{~nm}$ and $1.2 \mathrm{~nm}$, respectively, are evident. Finally, it is worth noting that all the spectra in Figs 15(d)-15(f) exhibit side lobes around the main peaks. These lobes could be attributed to the coupling of the core mode into asymmetric cladding modes probably due to not-perfect symmetry of arcinduced perturbation. Ivanov and Rego demonstrated that the asymmetry in arc perturbation is principally caused by the temperature gradient during arc discharge (Ivanov \& Rego, 2007).

\subsection{Applications of LPGs in PCFs}

As LPGs in standard fiber (see section 2) also LPGs in PCFs have found many promising applications for temperature, strain, bend, torsion, pressure, and biochemical sensors as well as they are becoming appealing for communications applications. In this section a brief review of the main applications of LPGs in PCFs is presented.

Concerning the temperature dependence of LPGs in IG-PCF, most of the papers report that temperature sensitivity is of the order of few picometers (up to 10) per degrees centigrade (Dobb et al., 2004; Petrovic et al., 2007; Zhu et al. 2005; Humbert et al., 2004) while the sensitivity of gratings in SMF is reported to be in the range $30-200 \mathrm{pm} /{ }^{\circ} \mathrm{C}$ or more (James \& Tatam, 2003; Rego et al. 2005b). The shifts of LPG bands due to thermal changes is principally attributed to two factors such as the thermooptic effect and the thermal expansion of the fiber that force wavelength shifts of opposite signs (Petrovic et al., 2007). As in SMFs, the impact of the former is dominant and determines the sign of the shift. The unique property of all silica-air fibers is that the light propagates mainly through the silica, and thus, the variations in the effective indices of the core and cladding modes with the refractive index of silica nearly cancel each other in the second term. The overall effect is a red wavelength shift of only a few picometers. This explains the very weak sensitivity of the LPG to temperature. The relatively low temperature sensitivity of the LPG in PCFs despite the sensitivity to other external parameters such as strain, bending and refractive index permitted to proposed several sensing configurations. Just to name a few of them, Dobb et al. (Dobb et al. 2004, 2006) demonstrated a temperature-insensitive LPG sensor to measure strain or curvature. The LPG structure with a period of $500 \mu \mathrm{m}$ was written by EAD. The LPG was characterized for temperature, strain and curvature and showed sensitivities of $0 \pm 10 \mathrm{pm} /{ }^{\circ} \mathrm{C},-2.04 \pm 0.12 \mathrm{pm} / \mu \varepsilon$, and $3.7 \mathrm{~nm} / \mathrm{m}$, respectively. Similar study has been conducted from on LPG fabricated by the use of focused $\mathrm{CO}_{2}$ laser beam (Y. Wang et al., 2006; C. Zhao et al., 2008). These structures written with $\mathrm{CO}_{2}$ laser were proposed as straininsensitive high-temperature PCF sensors ( $\mathrm{Zhu}$ et al., 2005). For high-temperature applications, LPGs written in PCF with the electric arc technique have also shown an adequate performance, as was reported in (Humbert et al. 2004). Wang et al. also proposed an dipper characterization of the strain and temperature sensitivity as function of the external grooves created by focused $\mathrm{CO}_{2}$ laser (Y. Wang, 2006). They realized two LPGs with 
same length and pitch but with different notches on the fiber surface: the first one presents evident grooves on the fiber surface achieved with setup provided in (Y. Wang, 2006), the second grating was fabricated by use of the same laser setup but a lower dosage of irradiation and presents no observable grooves on the fiber surface. The responses of the resonant wavelength to tensile strain and temperature of LPGs with and without visible notches is plotted in Fig. 16. The strain sensitivity of the LPG with grooves $(-7.6 \mathrm{pm} / \mu \varepsilon)$ is about 25 times higher than the LPG without physical deformation $(-0.31 \mathrm{pm} / \mu \varepsilon)$ (Fig. 16(a)) whereas the temperature sensitivities of the two LPGs are approximately the same (Fig. 16(b)). The asymmetrical structure caused by the periodic grooves introduces microbend when the LPG is axially stretched, which effectively enhanced the effective refractive index change of the LPGs with grooves. However the LPG with visible grooves also demonstrated very strong polarization dependent loss and can be used as in-fiber polarizers with good temperature stability (Y. Wang, 2007).
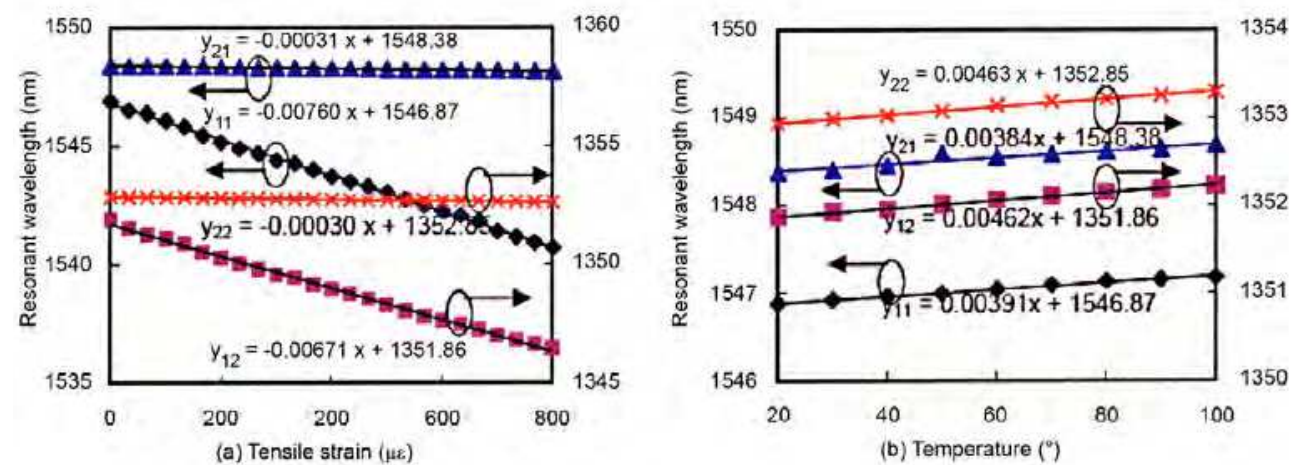

Fig. 16. Resonant wavelength of LPFG via (a) the tensile strain and (b) the temperature: and $\mathbf{r e f e r}$ to LPG with period grooves and $\boldsymbol{\Delta}$ and X refer to LPG without period grooves. Reprinted from (Y. Wang et al. 2006) with permission of Optical Society of America.

Han et al. demonstrated simultaneous measurement of strain and temperature using LPGs written in PCFs with different air-hole sizes (Han et al., 2007). They fabricated LPGs in IGPCFs with Ge-doped core and different air-hole size by the UV exposure (at $244 \mathrm{~nm}$ ) through an amplitude metal mask. They experimentally proved that the strain sensitivity of LPFGs depend on the air-hole size. Since all fibers have the same material composition, the LPGs exhibited similar temperature sensitivities regardless of the air-hole size. However, the strain sensitivities of the LPGs are different because of the different cross-sectional areas of the fibers depending on the different air-hole sizes. Similar functionality was reported by Sun et al. with processing based on an artificial neural network (Sun et al, 2007). Pressure sensing using an LPG fabricated in a PCF was presented in (Lim et al. 2004). Later, a hydrostatic pressure sensor using a tapered LPG written in PCF by the electric arc technique was also reported (Bock et al., 2007). The pressure sensitivity was found to be $11.2 \mathrm{pm} / \mathrm{bar}$, a factor of two higher than the value found in standard single-mode fibers.

Concerning to the sensing for chemical application, LPGs in PCF seem an appealing technological platform. Spectral sensitivity to refractive index of the surround medium is possible only if part of guided light comes into contact with surrounding materials (Petrovic 
et al., 2007). On this topic Dobb et al. experimentally investigated the SRI sensitivity of LPGs written in different fibers by EAD (Dobb et al, 2006). They demonstrated that LPGs written in large mode area (LMA) PCFs could be used for sensing SRI whereas the no-LMA PCFs exhibit minimal changes to SRI. They declare the spectral response of former gratings is similar to the behavior of LPGs fabricated in SMFs. The sensitivity increases as the SRI approaches that of the cladding. Besides, when the SRI and cladding indices are matched, the cladding appears to be infinite and thus, no cladding modes are supported. However the most appealing characteristic of the PCFs is to provide strong light/material interaction inside the fiber-hole, which offers a new features for developing ultra-sensitive and distributed gas and liquid sensors. Rindorf et al. presented LPG in photonic crystal fibers as sensitive biochemical sensor (Rindorf et al., 2006). A layer of biomolecules was immobilized on the sides of the holes of the PCF and by observing the shift in the resonant wavelength of the LPG it was possible to measure the thickness of the layer. The thicknesses of a monolayer of poly-L-lysine and double-stranded DNA was measured with s sensitivity of approximately $1.4 \mathrm{~nm} / 1 \mathrm{~nm}$. Later, LPG in large-mode-area PCF was presented as highly sensitive refractometers exhibiting $1500 \mathrm{~nm} /$ RIU at a refractive index of 1.33 (Rindorf and Bong, 2008). The high sensitivity is obtained by infiltrating the sample into the holes of the PCF to give a strong interaction between the sample and the probing field.

Furthermore, as for the same device in SMF, LPGs in PCF have found useful applications in optical communications systems. For example, a tuneable long-period grating filter in a hybrid polymer-silica PCF was reported in 2002 (Kerbage et al., 2002). The polymer is infused into the fiber holes and it is possible to change the LPG resonance wavelength in a range of $200 \mathrm{~nm}$, with a temperature variation of $10^{\circ} \mathrm{C}$. Recently, by utilizing coupled-mode theory, a design of band stop filter based on optimal LPG parameters (in terms of full-width half-maximum and grating length) in PCF is presented (Seraji et al., 2011). The analysis is presented for optimization of LPG length and number of gratings with respect to air-hole spacing $\Lambda$, hole diameter $d$, and air filling factor $d / \Lambda$ of the PCF in which LPG is inscribed. It is worth noting that also the response of LPGs in HC-PCF as function of physical parameters changes in terms of temperature stain and bending was investigated. The responses of the first LPG in the HC-PCF realized by focused $\mathrm{CO}_{2}$ laser to temperature, strain and bend in reported in Fig. 17 (Y. Wang at al., 2008). The temperature sensitivity of the resonant wavelength is about $2.9 \mathrm{pm} /{ }^{\circ} \mathrm{C}$. Accordingly to LPG in IG-PCF, the wavelength sensitivity is one to two orders of magnitude less than those of the LPGs in SMFs (Bhatia \& Vengsarkar, 1996). Also, the same LPG (in HC-PCF) was immerged into liquids with different refractive index and the resonant wavelength hardly changed, whereas the LPGs in SMFs are very sensitive to SRI. Differently, with the increase of applied tensile strain, the resonant wavelength of $\mathrm{CO}_{2}$ LPG shifted linearly toward shorter wavelength with a strain sensitivity of $-0.83 \mathrm{~nm} / \mathrm{m} \varepsilon$ and thus with sensitivity higher than that typical of LPGs in SMFs (Bhatia \& Vengsarkar, 1996). Finally, when the curvature of LPG was increased to 13.3m-1, the resonant wavelength changed by only $\pm 8 \mathrm{pm}$. From these results Wang et al. declare that LPG in HC-PCF achieved by $\mathrm{CO}_{2}$ laser may be used as a strain sensor without crosssensitivity to temperature, curvature, and external refractive index.

Finally, Fig. 18 reports the resonant wavelengths of the LPG in HC-PCF realized by EAD as function of the temperate. The grating take into consideration for the thermal characterization is composed by 25 perturbation with pitch of $\Lambda=400 \mu \mathrm{m}$. The spectrum of this grating is presented in Fig. 15(b) showing two attenuation bands at $1495.4 \mathrm{~nm}$ and $1520.3 \mathrm{~nm}$, respectively (Iadicicco et al., 2011). Thermal characterization in range $30-80{ }^{\circ} \mathrm{C}$ 


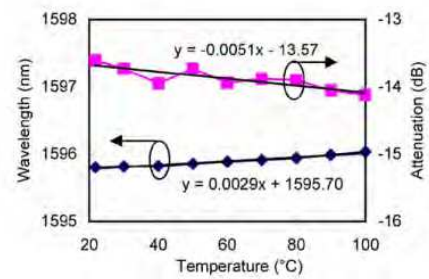

a)

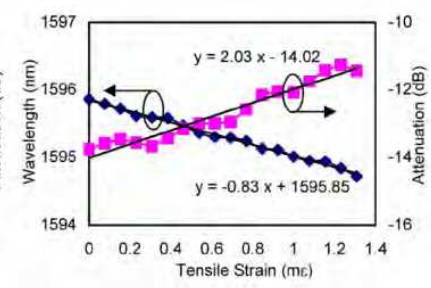

b)

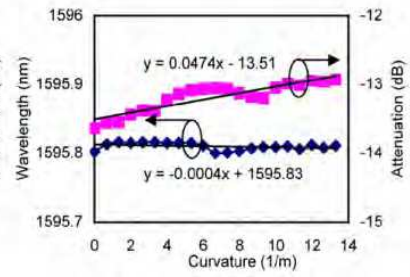

c)

Fig. 17. Measured resonant wavelength and peak transmitted attenuation of the LPG in HCPCF as functions of a) temperature, b) tensile strain and c) curvature. Reprinted from ( $Y$. Wang et al., 2008) with permission of Optical Society of America.
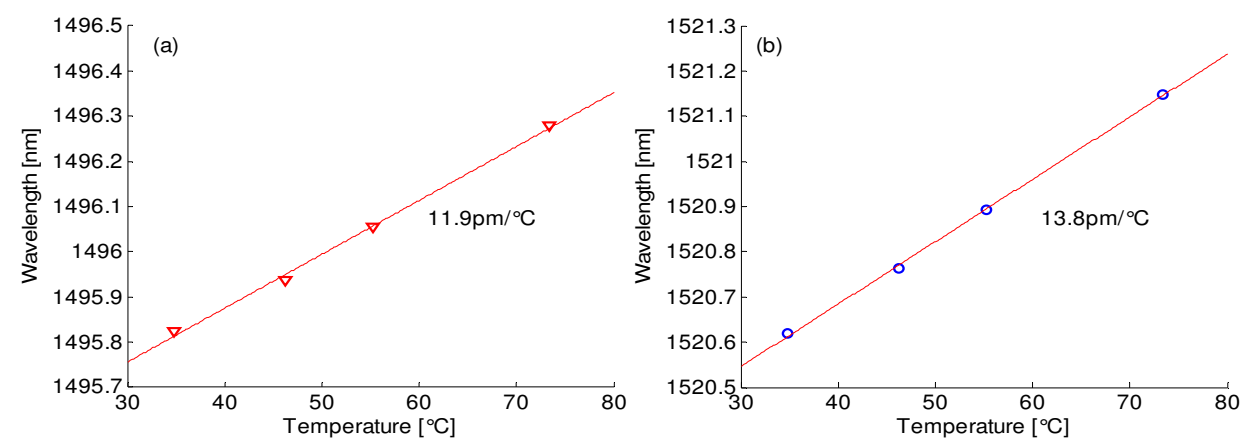

Fig. 18. Measured resonant wavelengths of the LPG in HC-PCF achieved by EAD as functions of temperature for left (a) and right (b) band.

are obtained using a furnace and a commercial FBG-based temperature sensor as reference. Accordingly with other LPG prototype above presented, both attenuation bands exhibit red shift with temperature increasing. Sensitivity of $11.9 \mathrm{pm} /{ }^{\circ} \mathrm{C}$ (Fig. $18(\mathrm{a})$ ) and $13.8 \mathrm{pm} /{ }^{\circ} \mathrm{C}$ (Fig. 18(b)) are measured for the left and right band respectively. In addition, the same LPG was characterized to different SRIs by using different liquids with RI ranging from 1.33 to 1.47 . As expected from the HC-PCFs mode fields distribution, no spectral changes were measured. Compared with the LPG in HC-PCF fabricated by focused $\mathrm{CO}_{2}$ laser (Y. Wang et al., 2008), it exhibits higher sensitivity versus thermal changes even if it is kept significantly lower than LPGs in SMFs (Bhatia et al, 1996). Besides, accordingly with $\mathrm{CO}_{2}$ laser, EAD based LPG exhibits trivial sensitivity to SRI.

In conclusion the LPGs in PCF (both IG and principally HC) act as novel platform to develop future devices with high performance principally for sensing applications. However at the state of the art, they still represent a very young technology requiring more work before of the industrialization of full sensing systems based on LPGs in PCF.

\section{Conclusions}

In this work, the fabrication techniques and application fields of LPGs in new generation optical fibers involving D-shaped fiber and solid- and hollow-core PCFs are reported. Despite the mature status of LPG technology in SMFs, in fact, LPGs transfer into new 
hosting fiber with novel features in terms of tuning and sensitivity of the guided field to environmental parameters is not easy. However, to address this issue, a lot of work has been made in the last decade and further work is currently in progress, since LPGs in new generation optical fibers seem to be extremely promising technological platforms.

D-shaped fiber seems the easiest solution to enhance the interaction between the guided mode and the surrounding medium. Among the different configurations, highly attractive is the evanescent-wave LPG made by patterning HRI layer on etched D-fiber surface and exhibiting (in a not optimized prototype) SRI sensitivity around the water RI of $\sim 700$ $\mathrm{nm} /$ RIU. From these results, evanescent-wave LPGs represent an extremely attractive technological platform for chemical and/or biological sensing. In addition, the possibility to use patterned coating with different properties (electro-optical, magneto-optical, etc.) to fabricate such devices would open the way to self-functionalized evanescent-wave LPGs suitable for specific applications.

Furthermore also LPGs in PCFs act as a novel technological platform for the development of optical fiber devices. It is important to distinguish between different PCFs. LPGs in IG-PCFs are in our opinion a quite-mature technology. Several approaches have been adopted to fabricate grating in IG-PCFs and many works report on their sensing capabilities, ranging from chemical/biological applications to physical parameters detections. On the other side, only few prototypes of LPGs in HC-PCFs have been reported. However we believe that the last configuration represents the most attractive one for chemical/biological applications in light of the strong light/material interaction that HC fibers allow inside the fiber hollow core. On this topic more work is required and it is reasonable to believe that good results will be published in next years

\section{References}

Allsop, T., Gillooly, A., Mezentsev, V., Earthgrowl-Gould, T., Neal, R., Webb, D. J. \& Bennion, I. (2004). Bending and orientational characteristics of long period gratings written in D-shaped optical fiber. IEEE Transactions on Instrumentation and Measurement, Vol.53, No.1, (February 2004), pp. 130-135, ISSN 0018-9456

Allsop, T., Dobb, H., Mezentsev, V., Earthgrowl, T., Gillooly, A., Webb, D. J. \& Bennion, I. (2006). The spectral sensitivity of long-period gratings fabricated in elliptical core D-shaped optical fibre. Optics Communications, Vol.259, No.2, (March 2006), pp. 537544, ISSN 0030-4018

Allsop, T., Kalli, K., Zhou, K., Laia, Y., Smith, G., Dubov, M., Webb, D. J., \& Bennion, I. (2008). Long period gratings written into a photonic crystal fiber by a femtosecond laser as directional bend sensors. Optics Communications, Vol. 281, No. 20, October 2008, pp. (5092-5096), ISSN 0030-4018

Barnes, J. A., Brown, R. S., Cheung, A. H., Dreher, M. A., Mackey, G., \& Loock, H.-P. (2010). Chemical sensing using a polymer coated long-period fiber grating interrogated by ring-down spectroscopy. Sensors and Actuators B: Chemical, Vol. 148, No. 1, pp. 221226. ISSN 0925-4005

Bhatia V. (1999). Applications of long-period gratings to single and multi-parameter sensing. Opt. Express, Vol. 4, pp. 457-66, ISSN 1094-4087.

Bhatia V. \& Vengsarkar A.M. (1996). Optical fiber long-period grating sensors. Optics Letters, Vol. 21, pp. 692-694, ISSN 1539-4794. 
Birks, T. A., Roberts, P. J., Russell, P. S. J., Atkin, D. M., \& Shepherd, T. J. (1995). Full 2-D photonic bandgaps in silica/air structures. Electronics Letters, Vol. 31, No. 22, October 1995, pp. (1941-1943), ISSN 0013-5194

Block, U. L., Dangui, V., Digonnet, M. J. F., \& Fejer, M. M. (2006). Origin of Apparent Resonance Mode Splitting in Bent Long-Period Fiber Gratings. Journal of Lightwave Technology, Vol. 24, No. 2, pp. 1027-. ISSN 0733-8724

Bock W., Chen J., Mikulic P., Eftimov T., Korwin-Pawlowski M. (2007). Pressure sensing using periodically tapered long-period gratings written in photonic crystal fibers. Meas. Sci. Technol., Vol. 18, pp. 3098-3102, ISSN 1361-6501

Canning J. (2008). Fibre gratings and devices for sensors and lasers. Laser \& Photon. Rev., Vol. 2, No. 4, pp. 275-289. ISSN 1863-8899.

Chen, X., Zhou, K., Zhang, L. \& Bennion, I. (2004). Optical chemsensors utilizing longperiod fiber gratings UV-inscribed in D-fiber with enhanced sensitivity through cladding etching. IEEE Photonics Technology Letters, Vol.16, No.5, (May 2004), pp. 1352-1354, ISSN 1041-1135

Chen, X., Zhou, K., Zhang, L. \& Bennion, I. (2005). Simultaneous measurement of temperature and external refractive index by use of a hybrid grating in D fiber with enhanced sensitivity by HF etching. Applied Optics, Vol.44, No.2, (January 2005), pp. 178-182, ISSN 0003-6935

Chen, X., Zhou, K., Zhang, L., \& Bennion, I. (2007). Dual-peak long-period fiber gratings with enhanced refractive index sensitivity by finely tailored mode dispersion that uses the light cladding etching technique. Applied Optics, Vol. 46, No. 4, pp. 451-455. ISSN 1559-128X

Chiang, K. S., \& Liu, Q. (2006) Long-period gratings for application in optical communications, in Proc. 5th International Conference on Optical Communications and Networks and 2nd International Symposium on Advances and Trends in Fiber Optics and Applications (ICOCN/ATFO 2006) (Chengdu, China, Sept. 2006), pp.128-133

Choi, H. Y., Park, K. S., \& Lee, B. H. (2008). Photonic crystal fiber interferometer composed of a long period fiber grating and one point collapsing of air holes. Optics Letters, Vol. 33, No. 8, 2008, pp. (812-814), ISSN 1539-4794

Chung, K.-W., \& Yin, S. (2004). Analysis of a widely tunable long-period grating by use of an ultrathin cladding layer and higher-order cladding mode coupling. Optics Letters, Vol. 29, No. 8, pp. 812-814. ISSN 0146-9592

Corres, J. M., del Villar, I., Matias, I. R., \& Arregui, F. J. (2007). Fiber-optic pH-sensors in long-period fiber gratings using electrostatic self-assembly. Optics Letters, Vol. 32, No. 1, pp. 29-31. ISSN 0146-9592

Cusano, A., Iadicicco, A., Pilla, P., Contessa, L., Campopiano, S., Cutolo, A., \& Giordano, M. (2005). Cladding mode reorganization in high-refractive-index-coated long-period gratings: effects on the refractive-index sensitivity. Optics Letters, Vol. 30, No. 19, pp. 2536-2538. ISSN 0146-9592

Cusano A., Iadicicco A., Pilla P., Contessa L., Campopiano S., Cutolo A., Giordano M. (2006a). Mode Transition in High Refractive Index Coated Long Period Gratings. Optics Express, Vol. 14, No. 1, pp. 19-34, ISSN 1094-4087.

Cusano, A., Iadicicco, A., Pilla, P., Contessa, L., Campopiano, S., Cutolo, A., Giordano, M., \& Guerra, G. (2006b). Coated Long-Period Fiber Gratings as High-Sensitivity 
Optochemical Sensors. Journal of Lightwave Technology, Vol. 24, No. 4, pp. 1776-. ISSN 0733-8724

Cusano, A., Iadicicco, A., Paladino, D., Campopiano, S., Cutolo, A. \& Giordano, M. (2007). Micro-structured fiber Bragg gratings. PartII: towards advanced photonic devices. Optical Fiber Technology, Vol.13, No.4, (October 2007), pp. 291-301, ISSN 1068-5200

Cusano, A.; Paladino, D.; Iadicicco, A. (2009a). Microstructured Fiber Bragg Gratings. Journal of Lightwave Technology, Vol. 27, No. 11, pp. 1663 - 1697. ISSN 0733-8724

Cusano, A., Pilla, P., Giordano, M. \& Cutolo, A. (2009b). Modal transition in nano-coated long period fiber gratings: principle and applications to chemical sensing, In: Advanced Photonic Structures for Biological and Chemical Detection, X. Fan, (Ed.), 35-76, Springer, ISBN 978-0-387-98060-7, Dordrecht, Heidelberg, London, New York

Del Villar, I., Matias, I., Arregui, F., \& Lalanne, P. (2005). Optimization of sensitivity in Long Period Fiber Gratings with overlay deposition. Optics Express, Vol. 13, No. 1, pp. 5669. ISSN 1094-4087

DeLisa, M. P., Zhang, Z., Shiloach, M., Pilevar, S., Davis, C. C., Sirkis, J. S., \& Bentley, W. E. (2000). Evanescent Wave Long-Period Fiber Bragg Grating as an Immobilized Antibody Biosensor. Analytical Chemistry, Vol. 72, No. 13, pp. 2895-2900. ISSN 00032700

Diez, A., Birks, T.A., Reeves, W.H., Mangan, B.J., \& Russell, P.St.J. (2000). Excitation of cladding modes in photonic crystal fibers by flexural acoustic waves, Optics Letters, Vol. 25 ,No. 20, 2000, pp. (1499-1501), ISSN 1539-4794

Dobb H., Kalli K., \& Webb D. J. (2004). Temperature-insensitive long-period grating sensors in photonic crystal fiber. Electron. Lett., Vol. 40, pp. 657-658, ISSN 0013-5194.

Dobb, H., Kalli, K., \& Webb, D. J., (2006). Measured sensitivity of arc-induced long-period grating sensors in photonic crystal fibre. Optics Communications, Vol. 260, No 1, April 2006,pp. (184-191), ISSN 0030-4018

Eggen, C. L., Lin, Y. S., Wei, T., \& Xiao, H. (2010). Detection of lipid bilayer membranes formed on silica fibers by double-long period fiber grating laser refractometry. Sensors and Actuators B: Chemical, Vol. 150, No. 2, pp. 734-741. ISSN 0925-4005

Eggleton, B.J., Westbrook, P.S., Windeler, R.S., Spalter, S., \& Strasser, T.A. (1999). Grating resonances in air silica microstructured optical fiber. Optics Letters, Vol. 24 , No. 21, 1999, pp. (1460-1462), ISSN 1539-4794

Erdogan T. (1997). Fiber Grating Spectra. J. of Lightwave Technology, Vol. 15, No. 8, pp. 12771295, ISSN 0733-8724

Espindola, R.P., Windeler, R.S., Abramov, A.A., Eggleton, B.J., Strasser, T.A. \& Di Giovanni, D.J. (1999). External refractive index insensitive air-clad long-period fiber grating. Electronics Letters, Vol. 35, No. 4, February 1999, pp. (327-328), ISSN 0013-5194

Falate, R., Kamikawachi, R. C., Müller, M., Kalinowski, H. J., \& Fabris, J. L. (2005). Fiber optic sensors for hydrocarbon detection. Sensors and Actuators B: Chemical, Vol. 105, No. 2, pp. 430-436. ISSN 0925-4005

Falciai, R., Mignani, A. G., \& Vannini, A. (2001). Long period gratings as solution concentration sensors. Sensors and Actuators B: Chemical, Vol. 74, No. (1-3), pp. 74-77. ISSN 0925-4005

Fotiadi, A. A., Brambilla, G., Ernst, T., Slattery, S. A., \& Nikogosyan, D. N., (2007). TPAinduced long-period gratings in a photonic crystal fiber: Inscription and 
temperature sensing properties. Journal of Optical Society of America B, Vol. 24, No. 7, Jul. 2007, pp. (1475-1481), ISSN 1520-8540

Frazao, O., Santos, J.L., Araujo, F. M., \& Ferreira, L.A., (2008). Optical sensing with photonic crystal fibers. Laser \& Photonics Reviews, Vol. 2, No. 6, November 2008, pp.(449-459), ISSN 1863-8899

Fu, M.-Y., Lin, G.-R., Liu, W.-F., Sheng, H.-J., Su, P.-C. \& Tien, C.-L. (2009). Optical fiber sensor based on air-gap long-period fiber gratings. Japanese Journal of Applied Physics, Vol.48, No.12, (December 2009), 120211 (3 pages), ISSN 0021-4922

Fu, M.-Y., Lin, G.-R., Liu, W.-F. \& Wu, C.W. (2011). Fiber-optic humidity sensor based on an air-gap long period fiber grating. Optical Review, Vol.18, No.1, (January 2011), pp. 93-95, ISSN 1340-6000

Gibson, R., Kvavle, J., Selfridge, R. \& Schultz, S. (2007). Improved sensing performance of Dfiber/planar waveguide couplers. Optics Express, Vol.15, No.5, (March 2007), pp. 2139-2144, ISSN 1094-4087

Gordon, J. D., Lowder, T. L., Selfridge, R. H. \& Schultz, S. M. (2007). Optical D-fiber-based volatile organic compound sensor. Applied Optics, Vol.46, No.32, (November 2007), pp. 7805-7810, ISSN 0003-6935

Gu, Z., Xu, Y., \& Gao, K. (2006). Optical fiber long-period grating with solgel coating for gas sensor. Optics Letters, Vol. 31, No. 16, pp. 2405-2407. ISSN 0146-9592

Han Y-G, Song S., Kim G.H., Lee K., Lee S., Lee J.H., Jeong C.H., Oh C.H., \& Kang H.J., (2007). Simultaneous independent measurement of strain and temperature based on long-period fiber gratings inscribed in holey fibers depending on air-hole size Optics Letters, Vol. 32, pp. 2245-2247, ISSN 1539-4794.

He, Y.-J., Lo, Y.-L., \& Huang, J.-F. (2006). Optical-fiber surface-plasmon-resonance sensor employing long-period fiber gratings in multiplexing. Journal of the Optical Society of America B, Vol. 23, No. 5, pp. 801-811. ISSN 0740-3224

He, Z., Tian, F., Zhu, Y., Lavlinskaia, N., \& Du, H. (w.d.). Long-period gratings in photonic crystal fiber as an optofluidic label-free biosensor. Biosensors and Bioelectronics, In Press, Corrected Proof. ISSN 0956-5663

Hong, K. S., Park, H. C., Kim, B. Y., Hwang, I. K., Jin, W. Ju, J., \& Yeom, D. I. (2008). 1000 nm tunable acousto-optic filter based on photonic crystal fiber. Applied Physics Letters, Vol. 92, No. 3, pp. 031110-1-031110-3, ISSN 1077-3118

Humbert, G., Malki, A., Fevrier, S., Roy, P., \& Pagnoux, D. (2003), Electric arc-induced longperiod gratings in Ge-free air-silica microstructure fibres. Electronics Letters, Vol. 39, No. 4, February, pp. (349-350), ISSN 0013-5194

Humbert, G., Malki, A., Fevrier, S., Roy, P., \& Pagnoux, D. (2004). Characterizations at high temperatures of long-period gratings written in germanium-free air-silica microstructure fiber. Opt. Lett., Vol. 29, pp. 38-40, ISSN 1539-4794.

Iadicicco A., Campopiano S., Giordano M., Cusano A. (2007). Spectral behavior in thinned long period gratings: effects of fiber diameter on refractive index sensitivity. Applied Optics, Vol. 46, p. 6945-6952, ISSN: 0003-6935.

Iadicicco A., Campopiano S., Cutolo A., Korwin-Pawlowski M.L., Bock W.J., CusanoA. (2008). Refractive Index Sensitivity in Thinned UV and Arc Induced Long-Period Gratings: A Comparative Study. International Journal on Smart Sensing and Intelligent Systems, Vol. 1, p. 354-369, 2008, ISSN: 1178-5608. 
Iadicicco A., Campopiano S., \& Cusano A. (2011a). Long Period Gratings in Hollow Core Fibers by Pressure Assisted Arc Discharge Technique. Photonic Technology Letters, Vol. pp, No 99 (in press - online available), ISSN 1041-1135.

Iadicicco A., Campopiano S., Cutolo A., \& Cusano A. (2011b). Long Period Grating in Hollow Core Fibers: Fabrication and Characterization. IEEE Sensors Conference, (in press).

Iredale, T.B.; Steinvurzel, P.; Eggleton, B.J., (2006). Electric-arc-induced long-period gratings in fluid-filled photonic bandgap fibre, Electronics Letters, Vol 42, No 13, pp 739 - 740, ISSN: 0013-5194

Ivanov O.V.\& RegoG. (2007). Origin of coupling to antisymmetric modes in arc-induced long-period fiber gratings. Opt. Express, Vol. 15, pp. 13936-13941, ISSN 1094-4087.

James, S. W., \& Tatam, R. P. (2003). Optical fibre long-period grating sensors: characteristics and application. Measurement Science and Technology, Vol. 14, No. 5, pp. R49-R61. ISSN 0957-0233

Jang, H. S., Park, K. N., Kim, J. P., Sim, S. J., Kwon, O. J., Han, Y.-G. \& Lee, K. S. (2009). Sensitive DNA biosensor based on a long-period grating formed on the sidepolished fiber surface. Optics Express, Vol.17, No.5, (March 2009), pp. 3855-3860, ISSN 1094-4087

Jiang, M., Zhang, A. P., Wang, Y.-C., Tam, H.-Y., \& He, S. (2009). Fabrication of a compact reflective long-period grating sensor with a cladding-mode-selective fiber end-face mirror. Optics Express, Vol. 17, No. 20, pp. 17976-17982. ISSN 1094-4087

Jin L., Jin W., Ju J., \& Wang Y. (2011). Investigation of Long-Period Grating Resonances in Hollow-Core Photonic Bandgap Fibers. J. Lightwave Technol., Vol. 29, pp. 1707-1713, ISSN 0733-8724.

Ju J., Jin W., \& Demokan M. S. (2004). Two-mode operation in highly birefringent photonic crystal fiber, IEEE Photon. Technol. Lett., Vol. 16, pp. 2472-2474, ISSN 1041-1135.

Ju, J., Jin, W., \& Ho, H. L. (2008). Compact in-fiber interferometer formed by long-period gratings in photonic crystal fiber. IEEE Photonics Technology Letters, Vol. 20, No. 23, December 2008, pp. (1899- 1901), ISSN 1041-1135

Ju, J., \& Jin, W. (2011). Long Period Gratings in Photonic Crystal Fibers. Photonic Sensors, DOI: 10.1007/s13320-011-0020-9, ISSN 2190-7439

Kaiser P.V., Astle H.W. (1974). Low-loss single material fibers made from pure fused silica. Bell System Technical Journal, Vol. 53, No. 6, pp. 1021-1039. ISSN 1538-7305.

Kakarantzas, G., Birks, T.A., \& Russell, P.St.J. (2002). Structured long-period gratings in photonic crystal fiber. Optics Letters, Vol. 27, No. 12, June 2002, pp. (1013-1015), ISSN 1539-4794

Kalachev, A. I., Nikogosyan, D. N., \& Brambilla, G. (2005). Long-period fiber grating fabrication by high-intensity femtosecond pulses at $211 \mathrm{~nm}$. Journal of Lightwave Technology, Vol. 23, No. 8, pp. 2568- 2578. ISSN 0733-8724

Kashyap R. (1999). Fiber Bragg Gratings, San Diego: Academic Press. ISBN 0124005608, 9780124005600

Kerbage C, Steinvurzel P., Hale A., Windeler R.S., Eggleton B.J. (2002). Microstructured optical fiber with tunable birefringence. Electron. Lett., Vol. 38, pp. 310-312, ISSN 0013-5194. 
Kersey A.D., Davis M.A., Patrick H.J., LeBlanc M., Koo K.P.,. Askins C.G, Putnam M.A., \& Friebele E.J.. (1997). Fiber Grating Sensors. Journal of Lightwave Technology, Vol. 15, No. 8, pp. 1442- 1463. ISSN 0733-8724.

Kim, D. W., Zhang, Y., Cooper, K. L., \& Wang, A. (2006). Fibre-optic interferometric immuno-sensor using long period grating. Electronics Letters, Vol. 42, No. 6, pp. 324325. ISSN 0013-5194

Kim, H.-J., Kwon, O.-J., Han, Y.-G., Lee, M. K. \& Lee, S. B. (2010). Surface long-period fiber gratings inscribed in photonic crystal fibers. Journal of the Korean Physical Society, Vol.57, No.6, (December 2010), pp. 1956-1959, ISSN 0374-4884

Kim, H.-J., Kwon, O.-J., Lee, S. B. \& Han, Y.-G. (2011). Measurement of temperature and refractive index based on surface long-period gratings deposited onto a D-shaped photonic crystal fiber. Applied Physics B: Lasers and Optics, Vol.102, No.1, (January 2011), pp. 81-85, ISSN 0946-2171

Knight, J. C., Birks, T. A., Russell, P. S. J. , \& Atkin, D. M.. 1996. All-silica single-mode optical fiber with photonic crystal cladding. Optics Letters, Vol. 21, No. 19, 1996, pp. 15471549. ISSN 539-4794

Konstantaki, M., Pissadakis, S., Pispas, S., Madamopoulos, N., \& Vainos, N. A. (2006). Optical fiber long-period grating humidity sensor with poly(ethylene oxide)/cobalt chloride coating. Applied Optics, Vol. 45, No. 19, pp. 4567-4571. ISSN 1559-128X

Kuhlmey B.T., Eggleton B.J., \& Wu D.K.C. (2009). Fluid-Filled Solid-Core Photonic Bandgap Fibers. IEEE Journal of Lightwave Techonology, Vol. 27, pp. 1617-1630, ISSN 0733-8724.

Kumar, A. \& Varshney, R. K. (1984). Propagation characteristics of highly elliptical core optical waveguides: a perturbation approach. Optical and Quantum Electronics, Vol.16, No.4, (July 1984), pp. 349-354, ISSN 0306-8919

Lee, D., Jung, Y., Jeong, Y. S., Oh, K., Kobelke, J., Schuster, K., \& Kirchhof, J. (2006). Highly polarization-dependent periodic coupling in mechanically induced long period grating over air-silica fibers. Optics Letters, Vol. 31, No. 3, 2006, pp. (296298), ISSN 1539-4794

Lee, H. W., \& Chiang, K. S. (2009). $\mathrm{CO}_{2}$ laser writing of long-period fiber grating in photonic crystal fiber under tension. Optics Express, Vol. 17, Issue 6, March 2009, pp. (45334539), ISSN 1094-4087

Lee, J., Chen, Q., Zhang, Q., Reichard, K., Ditto, D., Mazurowski, J., Hackert, M., \& Shizhuo, Y. (2007). Enhancing the tuning range of a single resonant band long period grating while maintaining the resonant peak depth by using an optimized high index indium tin oxide overlay. Applied Optics, Vol. 46, No. 28, pp. 6984-6989. ISSN 1559$128 \mathrm{X}$

Lim, J.H., Lee, K.S., Kim, J.C., \& Lee, B.H. (2004). Tunable fiber gratings fabricated in photonic crystal fiber by use of mechanical pressure, Optics Letters, Vol. 29, No. 4, February 2004, pp. (331-333), ISSN 1539-4794

Liu Y., Wang L., Zhang M., Tu D., Mao X., \& Liao. Y. (2007). Long-Period Grating Relative Humidity Sensor With Hydrogel Coating. IEEE Photonics Technology Letters, Vol. 19, No. 12, pp. 880-882. ISSN 1041-1135

Liu, S., Jin, L., Jin, W., Wang, Y., \& Wang, D. N. (2010). Fabrication of Long-Period Gratings by Femtosecond Laser-Induced Filling of Air-Holes in Photonic Crystal Fibers. IEEE Photonics Technology Letters, Vol. 22, No. 22, November 2010, pp. (1635-1637), ISSN 1041-1135 
Morishita, K. \& Miyake, Y. (2004). Fabrication and resonance wavelengths of long-period gratings written in a pure-silica photonic crystal fiber by the glass structure change. Journal of Lightwave Technology, Vol. 22, No. 1, 625-630, ISSN 0733-8724.

Mosquera, L., Sàez-Rodriguez, D., Cruz, J. L., \& Andrès, M. V. (2010). In-fiber Fabry-Perot refractometer assisted by a long-period grating. Optics Letters, Vol. 35, No. 4, pp. 613-615. ISSN 0146-9592

Ozcana A. \& Demircib U. (2007). Rewritable self-assembled long-period gratings in photonic bandgap fibers using microparticles. Optics Communications, Vol 270, No 2, pp 225228, ISSN 0030-4018

Parka, K. N., Erdogan, T., \& Lee, K. S., (2006). Cladding mode coupling in long-period gratings formed in photonic crystal fibers. Optics Communications, Vol. 266, No. 2, 2006, pp. (541-545), ISSN 0030-4018

Patrick, H. J., Kersey, A. D., \& Bucholtz, F. (1998). Analysis of the Response of Long Period Fiber Gratings to External Index of Refraction. Journal of Lightwave Technology, Vol. 16, No. 9, pp. 1606-. ISSN 0733-8724

Petrovic J., Dobb H., Mezentsev V. K., Kalli K., Webb D. J., \& BennionI. (2007). Sensitivity of LPGS in PCFs fabricated by an electric arc to temperature, strain and external refractive index. Journal of Lightwave, Technol., Vol. 25, pp. 1306-1312. ISSN 07338724

Petrovic J. (2008). Modelling of Long Period Gratings in Photonic Crystal Fibres and Sensors Based on Them. InTech Book Modelling and Simulation, Chapter 22, 417, ISBN 978-3902613-25-7.

Pilla, P., Foglia Manzillo, P., Giordano, M., Korwin-Pawlowski, M. L., Bock, W. J., \& Cusano, A. (2008). Spectral behavior of thin film coated cascaded tapered long period gratings in multiple configurations. Optics Express, Vol. 16, No. 13, pp. 9765-9780. ISSN 1094-4087

Pilla, P., Manzillo, P. F., Malachovska, V., Buosciolo, A., Campopiano, S., Cutolo, A., Ambrosio, L., Giordano, M., \& Cusano, A. (2009). Long period grating working in transition mode as promising technological platform for label-free biosensing. Optics Express, Vol. 17, No. 22, pp. 20039-20050. ISSN 1094-4087

Quero, G., Crescitelli, A., Paladino, D., Consales, M., Buosciolo, A., Giordano, M., Cutolo, A. \& Cusano, A. (2011). Evanescent wave long-period fiber grating within D-shaped optical fibers for high sensitivity refractive index detection. Sensors and Actuators B: Chemical, Vol.152, No.2, (March 2011), pp. 196-205, ISSN 0925-4005

Rees, N. D., James, S. W., Tatam, R. P., \& Ashwell, G. J. (2002). Optical fiber long-period gratings with Langmuir?Blodgett thin-film overlays. Optics Letters, Vol. 27, No. 9, pp. 686-688. ISSN 0146-9592

Rego, G., Marques, P. V. S., Santos, J. L., \& Salgado, H. M. (2005a). Arc-Induced Long-Period Gratings. Fiber and Integrated Optics, Vol. 24, No. 3, pp. 245-259. ISSN 0146-8030

Rego G., Marques P.V.S., Salgado H.M., \& Santos J.L. (2005b). Simultaneous measurement of temperature and strain based on arc-induced long-period fibre gratings. Electron. Lett., Vol. 41, No. 2, pp. 60-62, Jan, ISSN 0013-5194.

Rego, G., Ivanov, O. V., \& Marques, P. V. S. (2006). Demonstration of coupling to symmetric and antisymmetric cladding modes in arc-induced long-period fiber gratings. Optics Express, Vol. 14, No. 21, pp. 9594-9599. ISSN 1094-4087 
Rindorf, L., Jensen, J. B., Dufva, M., Pedersen, L. H., Hoiby, P. E., \& Bang, O. (2006). Photonic crystal fiber long-period gratings for biochemical sensing. Optics Express, Vol. 14, No. 18, September 2008, pp.(8224-8231), ISSN 1094-4087

Rindorf L. \& Bang O. (2008). Highly sensitive refractometer with a photonic crystal-fiber long-period grating. Optics Letters, Vol. 33, pp. 563-564, , ISSN 1539-4794.

Russell R. (2003). Photonic crystal fibers. Science, Vol. 229, pp. 258-362. ISSN 1095-9203.

Seraji F.E., Farsinezhad S., \& Anzabi L.C. (2011). Optimization of long-period grating inscribed in large mode area photonic crystal fiber for design of band stop filter. Optik - International Journal for Light and Electron Optics, Vol. 122, N. 2011, pp. 58-62, ISSN 0030-4026.

Sharma, A., Kompella, J. \& Mishra, P. K. (1990). Analysis of fiber directional coupler half blocks using a new sinple model for single mode fiber. Journal of Lightwave Technology, Vol.8, No.2, (February 1990), pp. 143-151, ISSN 0733-8724

Shu, X., Zhang, L., \& Bennion, I. (2002). Sensitivity Characteristics of Long-Period Fiber Gratings. Journal of Lightwave Technology, Vol. 20, No. 2, pp. 255-. ISSN 0733-8724

Smietana, M., Bock, W. J., Mikulic, P., Ng, A., Chinnappan, R., \& Zourob, M. (2011). Detection of bacteria using bacteriophages as recognition elements immobilized on long-period fiber gratings. Optics Express, Vol. 19, No. 9, pp. 7971-7978. ISSN 10944087

Smith C.M., Venkataraman N., Gallagher M.T., Muller D., West J.A., Borrelli N.F.,. Allan D.C, \& Koch K.W. (2003). Low-loss hollow-core silica/air photonic bandgap fibre. Nature Vol. 424, pp. 657-659. ISSN: 0028-0836

Smith, K. H., Markos, D. J., Ipson, B. L., Schultz, S. M., Selfridge, R. H., Barber, J. P., Campbell, K. J., Monte, T. D. \& Dyott, R. B. (2004). Fabrication and analysis of a low-loss in-fiber active polymer waveguide. Applied Optics, Vol.43, No.4, (February 2004), pp. 933-939, ISSN 0003-6935

Smith, K. H. (2005). In-fiber optical devices based on D-fiber, Ph.D. Dissertation at Brigham Young University, April 2005, Available from http://contentdm.lib.byu.edu/ETD/image/etd738.pdf

Smith, K. H., Ipson, B. L., Lowder, T. L., Hawkins, A. R., Selfridge, R. H. \& Schultz, S. M. (2006). Surface-relief fiber Bragg gratings for sensing applications. Applied Optics, Vol.45, No.8, (March 2006), pp. 1669-1675, ISSN 0003-6935

Sun J., Chan C.C., Dong X.Y., \&. Shum. (2007). Application of an artificial neural network for simultaneous measurement of temperature and strain by using a photonic crystal fiber long-period grating. Meas. Sci. Technol. Vol. 18, pp. 2943-2948, ISSN 1361-6501.

Smith C.M., \& Venkataraman N, Gallagher M. T., Muller D., West J. A., Borrelli N.F., Allan D.C., \& Koch K.W. (2003). Low-loss hollow-core silica/air photonic bandgapfibre. Nature Vol. 424, pp. 657-659, 2003, ISSN 1476- 4687.

Steinvurzel P., Moore E.D., Mägi E.C., Kuhlmey B.T., \& Eggleton B.J. (2006a). Long period grating resonances in photonic bandgap fiber. Opt. Express, Vol. 14, No. 7, pp. 3007-3014, ISSN 1094-4087

Steinvurzel P., Moore E.D., Mägi E.C., \& Eggleton B.J. (2006b). Tuning properties of long period gratings in photonic bandgap fibers, Optics Letters., Vol. 31, No. 14, pp. 21032105, ISSN 1539-4794 
Tan, K. M., Tay, C. M., Tjin, S. C., Chan, C. C., \& Rahardjo, H. (2005). High relative humidity measurements using gelatin coated long-period grating sensors. Sensors and Actuators B: Chemical, Vol. 110, No. 2, pp. 335-341. ISSN 0925-4005

Tang, J.-L., Cheng, S.-F., Hsu, W.-T., Chiang, T.-Y., \& Chau, L.-K. (2006). Fiber-optic biochemical sensing with a colloidal gold-modified long period fiber grating. Sensors and Actuators B: Chemical, Vol. 119, No. 1, pp. 105-109. ISSN 0925-4005

Tien, C.-L., Hwang, C.-C., Liu, W.-F. \& Lin, T.-W. (2009a). Magnetic field sensor based on Dshaped long period fiber gratings, Proceedings of SPIE 7508 (2009 International Conference on Optical Instruments and Technology: Advanced Sesnor Technologies and Applications), 750811, ISBN 978-0-819-47894-8, Shanghai, China, October 19-21, 2009

Tien, C.-L., Lin, T.-W., Hsu, H.-Y. \& Liu, W.-F. (2009b). Double-sided polishing long period fiber grating sensors for measuring liquid refractive index, Proceedings of 2009 Asia Communications and Photonics (ACP) Conference and Exhibition, ISBN 978-1-55752877-3, Shanghai, China, November 2-6, 2009

Topliss, S. M., James, S. W., Davis, F., Higson, S. P. J., \& Tatam, R. P. (2010). Optical fibre long period grating based selective vapour sensing of volatile organic compounds. Sensors and Actuators B: Chemical, Vol. 143, No. 2, pp. 629-634. ISSN 0925-4005

Tripathi, S. M., Kumar, A., Marin, E. \& Meunier, J.-P. (2008). Side-polished optical fiber grating-based refractive index sensors utilizing the pure surface plasmon polariton. Journal of Lightwave Technology, Vol.26, No.13, (July 2008), pp. 1980-1985, ISSN 07338724

Tripathi, S. M., Marin, E., Kumar, A. \& Meunier, J.-P. (2009). Refractive index sensing characteristics of dual resonance long period gratings in bare and metal-coated Dshaped fibers. Applied Optics, Vol.48, No.31, (November 2009), pp. G53-G58, ISSN $1539-4522$

Tseng, S.-M. \& Chen, C.-L. (1992). Side-polished fibers. Applied Optics, Vol.31, No.18, (June 1992), pp. 3438-3447, ISSN 0003-6935

Vasil'ev, S. A., Medvedkov, O. I., Korolev, I. G., Bozhkov, A. S., Kurkov, A. S., \& Dianov, E. M. (2005). Fibre gratings and their applications. Quantum Electronics, Vol. 35, No. 12, pp. 1085-1103. ISSN 1063-7818

Vengsarkar A.M., Lemaire P.J., Judkins J.B., Bhatia V., Erdogan T., \& Sipe J.E., (1996). Longperiod fiber gratings as band-rejection filters. Journal of Lightwave Technology, Vol. 14, pp. 58-65, Jan, , ISSN 0733-8724

Venugopalan, T., Sun, T., \& Grattan, K. T. V. (2008). Long period grating-based humidity sensor for potential structural health monitoring. Sensors and Actuators A: Physical, Vol. 148, No. 1, pp. 57-62. ISSN 0924-4247

Wang Y., Xiao L., Wang D., \& Jin W. (2006). Highly sensitive long-period fiber-grating strain sensor with low temperature sensitivity. Optics Letters, Vol. 31, pp. 3414-3416, ISSN 1539-4794.

Wang, Y., Xiao, L., Wang, D. N., \& Jin, W. (2007). In-fiber polarizer based on a long-period fiber grating written on photonic crystal fiber. Optics Letters, Vol. 32, No. 9, May 2007, pp. (1035-1037), ISSN 1539-4794

Wang Y., Jin W., Ju J., Xuan H., Ho H.L., Xiao L., \& Wang D., (2008). Long period gratings in air-core photonic bandgap fibers, Opt. Exp., Vol. 16, No. 4, pp. 2784-2790, Feb, ISSN 1094-4087 
Wang Y. (2010). Review of long period fiber gratings written by CO2 laser. Journal of Applied Physics Vol. 108, No. 081101, pp. 081101-1 081101-18, ISSN 1089-7550

Wang, Z., Heflin, J. R., Stolen, R. H., \& Ramachandran, S. (2005). Highly sensitive optical response of optical fiber long period gratings to nanometer-thick ionic selfassembled multilayers. Applied Physics Letters, Vol. 86, No. 22, pp. 223104. ISSN 0003-6951

Wang, Z., Heflin, J. R., Van Cott, K., Stolen, R. H., Ramachandran, S., \& Ghalmi, S. (2009). Biosensors employing ionic self-assembled multilayers adsorbed on long-period fiber gratings. Sensors and Actuators B: Chemical, Vol. 139, No. 2, pp. 618-623. ISSN 0925-4005

Wei, X., Wei, T., Xiao, H., \& Lin, Y. S. (2008). Nano-structured Pd-long period fiber gratings integrated optical sensor for hydrogen detection. Sensors and Actuators B: Chemical, Vol. 134, No. 2, pp. 687-693. ISSN 0925-4005

West J. A., Smith C. M., Borrelli N. F., Allan D. C., \& Koch K. W. (2004). Surface modes in air-core photonic band-gap fibers. Opt. Express, Vol. 12, pp. 1485-1496, ISSN 10944087.

Xuewen S., Allsop, T., Gwandu, B., Lin Zhang, \& Bennion, I. (2001). High-temperature sensitivity of long-period gratings in B-Ge codoped fiber. IEEE Photonics Technology Letters, Vol. 13, No. 8, pp. 818-820. ISSN 1041-1135.

Yin, S., Chung, K.-W., \& Zhu, X. (2001). A novel all-optic tunable long-period grating using a unique double-cladding layer. Optics Communications, Vol. 196, No. 1-6, pp. 181186. ISSN 0030-4018

Zhao, C.-L., Xiao, L., Ju, J., Demokan M.S., \& Jin,W. (2008). Strain and temperature characteristics of a long-period grating written in a photonic crystal fiber and its application as a temperature-insensitive strain sensor. IEEE Journal of Lightwave Techonology, Vol. 26, No. 2, January 2008, pp. (220-227), ISSN 0733-8724.

Zhao, D., Zhou, K., Chen, X., Zhang, L., Bennion, I., Flockhart, G., MacPherson, W. N., Barton, J. S. \& Jones, J. D. C. (2004a). Implementation of vectorial bend sensors using long-period gratings UV-inscribed in special shape fibres. Measurement Science and Technology, Vol.15, No.8, (August 2004), pp. 1647-1650, ISSN 0957-0233

Zhao, D., Chen, X., Zhou, K., Zhang, L., Bennion, I., MacPherson, W. N., Barton, J. S. \& Jones, J. D. C. (2004b). Bend sensors with direction recognition based on longperiod gratings written in D-shaped fiber. Applied Optics, Vol.43, No.29, (October 2004), pp. 5425-5428, ISSN 0003-6935

Zhang, J., Tang, X., Dong, J., Wei, T., \& Xiao, H. (2008). Zeolite thin film-coated long period fiber grating sensor for measuring trace chemical. Optics Express, Vol. 16, No. 11, pp. 8317-8323. ISSN 1094-4087

Zhu Y., ShumP., Bay H.-W., Yan M., Yu X., Hu J., Hao J., \& Lu C. (2005). Strain-insensitive and high-temperature longperiod gratings inscribed in photonic crystal fiber. Opt. Lett., Vol. 30, pp. 367-369, ISSN 1539-4794. 


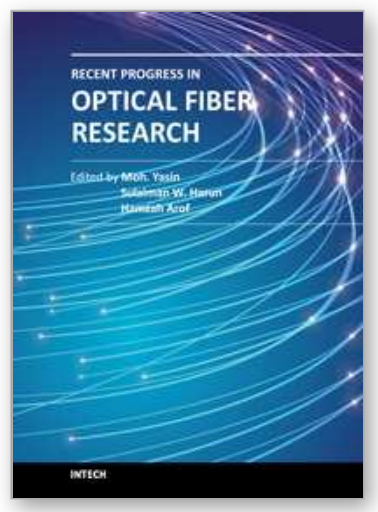

\author{
Recent Progress in Optical Fiber Research \\ Edited by Dr Moh. Yasin
}

ISBN 978-953-307-823-6

Hard cover, 450 pages

Publisher InTech

Published online 25, January, 2012

Published in print edition January, 2012

This book presents a comprehensive account of the recent progress in optical fiber research. It consists of four sections with 20 chapters covering the topics of nonlinear and polarisation effects in optical fibers, photonic crystal fibers and new applications for optical fibers. Section 1 reviews nonlinear effects in optical fibers in terms of theoretical analysis, experiments and applications. Section 2 presents polarization mode dispersion, chromatic dispersion and polarization dependent losses in optical fibers, fiber birefringence effects and spun fibers. Section 3 and 4 cover the topics of photonic crystal fibers and a new trend of optical fiber applications. Edited by three scientists with wide knowledge and experience in the field of fiber optics and photonics, the book brings together leading academics and practitioners in a comprehensive and incisive treatment of the subject. This is an essential point of reference for researchers working and teaching in optical fiber technologies, and for industrial users who need to be aware of current developments in optical fiber research areas.

\title{
How to reference
}

In order to correctly reference this scholarly work, feel free to copy and paste the following:

Agostino ladicicco, Domenico Paladino, Pierluigi Pilla, Stefania Campopiano, Antonello Cutolo and Andrea Cusano (2012). Long Period Gratings in New Generation Optical Fibers, Recent Progress in Optical Fiber Research, Dr Moh. Yasin (Ed.), ISBN: 978-953-307-823-6, InTech, Available from:

http://www.intechopen.com/books/recent-progress-in-optical-fiber-research/long-period-gratings-in-newgeneration-optical-fibers

\section{INTECH}

open science | open minds

\section{InTech Europe}

University Campus STeP Ri

Slavka Krautzeka 83/A

51000 Rijeka, Croatia

Phone: +385 (51) 770447

Fax: +385 (51) 686166

www.intechopen.com

\section{InTech China}

Unit 405, Office Block, Hotel Equatorial Shanghai

No.65, Yan An Road (West), Shanghai, 200040, China 中国上海市延安西路65号上海国际贵都大饭店办公楼405单元

Phone: +86-21-62489820

Fax: +86-21-62489821 
(C) 2012 The Author(s). Licensee IntechOpen. This is an open access article distributed under the terms of the Creative Commons Attribution 3.0 License, which permits unrestricted use, distribution, and reproduction in any medium, provided the original work is properly cited. 